Geom. Funct. Anal. Vol. 20 (2010) 1502-1547

DOI 10.1007/s00039-010-0096-1

Published online October 17, 2010

(C) 2010 The Author(s)

GAFA Geometric And Functional Analysis

This article is published with open access at Springerlink.com

\title{
A FINITARY VERSION OF GROMOV'S POLYNOMIAL GROWTH THEOREM
}

\author{
Yehuda Shalom And Terence TaO
}

\begin{abstract}
We show that for some absolute (explicit) constant $C$, the following holds for every finitely generated group $G$, and all $d>0$ :

If there is some $R_{0}>\exp \left(\exp \left(C d^{C}\right)\right)$ for which the number of elements in a ball of radius $R_{0}$ in a Cayley graph of $G$ is bounded by $R_{0}^{d}$, then $G$ has a finite-index subgroup which is nilpotent (of step $<C^{d}$ ). An effective bound on the finite index is provided if "nilpotent" is replaced by "polycyclic", thus yielding a non-trivial result for finite groups as well.
\end{abstract}

\section{Introduction}

A famous theorem of Gromov [Gr] asserts that all finitely generated groups of polynomial growth (thus, in the notation used below, one has $\left|B_{S}(R)\right| \leq R^{d}$ for some $d$ and all sufficiently large $R$, where $S$ is a fixed set of generators) are virtually nilpotent. This was generalized by van der Dries and Wilkie [DrW1] by assuming the polynomial growth condition $\left|B_{S}(R)\right| \leq R^{d}$ at an infinite number, rather than all scales. A second proof of this fact, which for the first time released the dependence on the involved solution to Hilbert's $5^{\text {th }}$ problem, was given recently by Kleiner [Kl], motivated by work of Colding-Minicozzi $[\mathrm{CM}]$ and using the theory of harmonic functions. In this paper, we refine Kleiner's work to obtain a further strengthening of Gromov's theorem that only requires the polynomial growth condition at one (sufficiently large, yet explicit) scale, and gives quantitative control on the nilpotency degree. To state the result (mentioned in the Abstract above) precisely, we need some notation.

DeFinition $1.1\left(\left(R_{0}, d\right)\right.$-growth groups). A finitely generated group is a pair $G=(G, S)$, where $G$ is a group, and $S \subset G$ is a finite non-empty symmetric set which generates $G$ (thus $S^{-1}:=\left\{s^{-1}: s \in S\right\}=S$ ). For each $g \in G$, we define $\|g\|_{S}:=\inf \left\{n: g \in S^{n}\right\}$ to be the length of the smallest word with alphabet $S$ that evaluates to $g$. For each $R>0$, we define $B_{S}(R):=\left\{g \in G:\|g\|_{S} \leq R\right\}$ to be the collection of words in $S$ of length at most $R$. For $R_{0}, d>0$, we define a $\left(R_{0}, d\right)$-growth group to be a finitely generated group $(G, S)$ such that

$$
\left|B_{S}\left(R_{0}\right)\right| \leq R_{0}^{d}
$$

Keywords and phrases: Polynomial growth, nilpotent groups, harmonic functions 2010 Mathematics Subject Classification: 20F65, 10F19, 10F69, 31C05 
REMARK 1.2. One can of course generalize (1) by replacing the right-hand side of $R_{0}^{d}$ by $C R_{0}^{d}$ for some additional parameter $C$, as is customary in the literature. But this would add a new parameter to an already complicated notational system and so we have chosen to drop this parameter, as one can partially simulate it by increasing $d$ slightly and assuming $R_{0}$ is large. Note that a finitely generated group $(G, S)$ has polynomial growth if and only if there exists a $d$ such that $(G, S)$ is a $\left(R_{0}, d\right)$-growth group for all sufficiently large $R_{0}$.

Definition 1.3 (Quantitative finite index). Let $R, K>0$. A finitely generated group $\left(G^{\prime}, S^{\prime}\right)$ is said to be a $(K, R)$-subgroup of $(G, S)$ if $G^{\prime}$ is a subgroup of $G$, $S^{\prime} \subset B_{S}(R)$, and $B_{S}(K+1) \subset B_{S}(K) \cdot B_{S^{\prime}}(K)$. If we drop the final condition $B_{S}(K+1) \subset B_{S}(K) \cdot B_{S^{\prime}}(K)$, we say that $G^{\prime}$ is a $(\infty, R)$-subgroup of $G$.

REMARK 1.4. Observe that if $\left(G^{\prime}, S^{\prime}\right)$ is a $(K, R)$-subgroup of $(G, S)$ then $B_{S}(r+1) \subset B_{S}(r) \cdot B_{S^{\prime}}(K)$ for all $r \geq K$, and on iterating this we conclude $G \subset B_{S}(K) \cdot G^{\prime} k$. In particular, $G^{\prime}$ is a finite-index subgroup of $G$ of index at most $\left|B_{S}(K)\right|$. Conversely, if $\left(G^{\prime}, S^{\prime}\right)$ has finite index in $(G, S)$, then we can write $G$ as a finite union of cosets $x_{1} \cdot G^{\prime}, \ldots, x_{m} \cdot G^{\prime}$ of $G^{\prime}$, and in particular one has the relations $e x_{i}=x_{j_{e, i}} g_{e, i}$ for all $1 \leq i \leq m, e \in S$, and some $1 \leq j_{e, i} \in m, g_{e, i} \in G^{\prime}$. If one then sets $R:=\sup \left\{\left\|e^{\prime}\right\|_{S}: e^{\prime} \in S^{\prime}\right\}$ and $K:=\sup \left\{\left\|x_{i}\right\|_{S}: 1 \leq i \leq m\right\} \cup\left\{\left\|g_{e, i}\right\|_{S^{\prime}}: 1 \leq\right.$ $i \leq m, e \in S\}$ we see that $\left(G^{\prime}, S^{\prime}\right)$ is a $(K, R)$-subgroup. Note, however, that this argument does not (and cannot) give effective bounds on $K, R$.

EXAMPLE 1.5. Let $m=2 p-1$ be a large odd number. Then the (additive) finitely generated group $(\mathbf{Z} / m \mathbf{Z},\{-2,+2\})$ is a $(2,2)$-subgroup of $(\mathbf{Z} / m \mathbf{Z},\{-1,+1\})$, while conversely, $(\mathbf{Z} / m \mathbf{Z},\{-1,+1\})$ is merely a $(2, p)$-subgroup of $(\mathbf{Z} / m \mathbf{Z},\{-2,+2\})$. The intuition here is that while $\{-2,+2\}$ does generate the element 1 , this is a "global" fact (relying on the parity of $m$ ) rather than a "local" one, and thus cannot be detected in the limit $p \rightarrow \infty$ if one is only allowed to perform a bounded number of group operations. Thus we see that this quantitative notion of finite index not only measures the index of $G^{\prime}$ in $G$, but also the relative position of $S$ and $S^{\prime}$. This kind of "practical" interpretation of abstract (and often trivial) group theoretic notions is necessary in order to perform the quantitative arguments in this paper properly.

REMARK 1.6. It is immediate that if $(G, S)$ is a $\left(R_{0}, d\right)$-growth group, and $\left(G^{\prime}, S^{\prime}\right)$ is a $\left(K, R_{0}^{\kappa}\right)$-subgroup of $(G, S)$ for some $0<\kappa<1$, then $\left(G^{\prime}, S^{\prime}\right)$ is a $\left(R_{0}^{1-\kappa}, d /(1-\kappa)\right)$ growth group. This is analogous to the obvious fact that any finite-index subgroup of a group of polynomial growth, remains of polynomial growth.

Definition 1.7 (Virtual nilpotency). Let $K, R, s, D \geq 1$. A finitely generated group $(G, S)$ is said to be $(s, D)$-nilpotent if $|S| \leq D$ and $G$ is nilpotent of step at most $s$ (i.e. every $s^{\prime}$-fold iterated commutator vanishes for $s^{\prime}>s$ ). A group $(G, S)$ is said to be $(K, R, s, D)$-virtually nilpotent if it contains a $(s, D)$-nilpotent $(K, R)$-subgroup $\left(G^{\prime}, S^{\prime}\right)$.

Theorem 1.8 (Quantitative Gromov theorem). Let $d, R_{0}>0$, and assume that

$$
R_{0} \geq \exp \left(\exp \left(C d^{C}\right)\right)
$$


for some sufficiently large absolute constant $C$. Then every $\left(R_{0}, d\right)$-growth group is $\left(K\left(R_{0}, d\right), K\left(R_{0}, d\right), C^{d}, C^{d}\right)$-virtually nilpotent for some $K\left(R_{0}, d\right)$ depending only on $R_{0}, d$.

In order to simplify the exposition somewhat, we do not give an effective bound for $K\left(R_{0}, d\right)$ in our arguments, relying instead on an ineffective compactness argument to establish its finiteness. However, it is possible to eradicate this compactness argument from the proof by standard "quantifier elimination" techniques, at the cost of making it substantially lengthier (and the final bound for $K\left(R_{0}, d\right)$ obtained is quite poor, of Ackermann type in $d$ ). On the other hand, the arguments do give an effective value for $C$ (it appears that $C=100$ works, even if for clarity we won't keep track of this aspect, in which no tightness is claimed). We discuss full effectivization issues in section 15, along with the following result:

Theorem 1.9 (Fully quantitative weak Gromov theorem). Let $d>0$ and $R_{0}>0$, and assume that

$$
R_{0} \geq \exp \left(\exp \left(C d^{C}\right)\right)
$$

for some sufficiently large absolute constant $C$. Then every $\left(R_{0}, d\right)$-growth group has a normal subgroup of index at most $\exp \left(R_{0}^{\exp \left(\exp \left(d^{C}\right)\right)}\right)$ which is polycyclic.

This result is in fact established along sections 6-10 (see Proposition 5.2 below), and can be recommended to the reader as a natural "resting point" along the way to the full proof of the main Theorem 1.8. It captures the quantitative outcome made out of Kleiner's approach, and avoids completely the Milnor-Wolf part of the proof, which is only made semi-quantitative here. Note also that a completely effective version of Theorem 1.8 is still available when the group is assumed torsion free; see Corollary 15.6.

We have the following immediate corollary of Theorem 1.8:

Corollary 1.10 (Slightly super-polynomial growth implies virtual nilpotency). Let $(G, S)$ be a finitely generated group such that

$$
\left|B_{S}(R)\right| \leq R^{c(\log \log R)^{c}}
$$

for some $R>1 / c$, where $c>0$ is a sufficiently small absolute constant. Then $G$ is virtually nilpotent.

REMARK 1.11. By using completely ineffective compactness arguments, it is well known that Gromov's theorem implies the existence of some super-polynomial growth function for which the corollary holds, although no such explicit function was known before. It has been proposed that the subradical growth type $\exp (C \sqrt{n})$ might work (see [G1], [LuM] for some results in this direction). It is also interesting to compare the situation with Segal's refutation [S] of a conjecture of Lubotzky, Pyber and Shalev [LuPS] concerning the growth type of the number of finite-index subgroups of a residually finite group (as a function of the index). It was shown in $[\mathrm{S}]$ that here no super-polynomial bound could yield the same characterization as the polynomial one, of being virtually solvable of finite rank (a remarkable result of Lubotzky, Mann and Segal [LuMS], which relies, among other things, on Lazard's deep p-adic analogue of Hilbert's $5^{\text {th }}$ problem [L]). 
The nilpotent group generated by Theorem 1.8 has step at most $C^{d}$, but an inspection of the proof shows that it also has Hirsch length (i.e. sum of torsionfree ranks of the abelian quotients in a grading) at most $C^{d}$. It is a standard computation (which also follows from the well known Bass-Guivarc'h formula for the growth of nilpotent groups), that nilpotent groups of Hirsch length $r$ and step $s$ have polynomial growth of order at most $O(r s)$. We thus conclude

Corollary 1.12 (Polynomial growth at one scale implies polynomial growth at all scales). Let $d>0$ and $R_{0}>0$, and assume that

$$
R_{0} \geq \exp \left(\exp \left(C d^{C}\right)\right)
$$

for some sufficiently large absolute constant $C$. Let $G$ be a $\left(R_{0}, d\right)$-growth group. Then one has

$$
\left|B_{S}(R)\right| \leq K R^{C^{d}}
$$

for all $R \geq 1$, where $K=K\left(R_{0}, d\right)$ depends only on $R_{0}, d$.

Thus, polynomial growth of order $d$ at one (moderately large) scale implies polynomial growth of order $O(1)^{d}$ at all subsequent scales. We do not know if this exponential loss in the polynomial growth degree is necessary.

1.13 Overview of proof. Our arguments broadly follow the strategy used by Kleiner $[\mathrm{Kl}]$ to prove Gromov's theorem, being based in particular on the study of harmonic functions on the Cayley graph associated to $(G, S)$. Kleiner's proof proceeded, roughly, along the following steps:

(i) If $G$ is infinite amenable (or is merely without property $(\mathrm{T})$ ), then it admits a fixed-point free affine action on a Hilbert space, one of its orbit maps being a Hilbert space valued non-constant Lipschitz harmonic function on $G$. After taking a section, this implies the existence of a scalar non-constant Lipschitz harmonic function.

(ii) If $G$ has polynomial growth, then the space of real valued Lipschitz (or fixed polynomial growth) harmonic functions on $G$ is finite-dimensional.

(iii) From (i) and (ii), an infinite group $G$ of polynomial growth admits a finitedimensional representation with infinite image, or equivalently, a normal subgroup $H$ whose quotient $G / H$ is an infinite linear group.

(iv) Any linear group of polynomial growth is virtually solvable.

(v) From (iii) and (iv), an infinite group $G$ of polynomial growth admits a normal subgroup $H$ with virtually solvable - or better yet virtually infinite cyclic quotient $G / H$.

(vi) The group $H$ obtained in (v) as the kernel of a $\mathbf{Z}$-target homomorphism of a finite-index subgroup of $G$ has slower growth, and thus (by induction) is virtually nilpotent. Hence $G$ is virtually solvable (in fact, polycyclic).

(vii) Any virtually polycyclic (or merely solvable) group of polynomial growth is virtually nilpotent.

The idea is then to make the proofs of the statements (i)-(vii) as elementary as possible, so that they may be made quantitative. Note that for these purposes, 
statements involving infinite objects must be re-proved in a finitary version, and in the fully effective results (e.g. Theorem 1.9) only finitely many elements of the group are actually involved. This is a rather unconventional difficulty in geometric group theory, where Gromov's theorem is a cornerstone. We next remark on our modification of these steps.

Statement (i) is made quantitative in section 6 . The proof given in $[\mathrm{Kl}]$ uses ultralimits and considerations related to Kazhdan's property ( $\mathrm{T}$ ), and is thus of a qualitative nature. However, it turns out that one can use the spectral theory of the Laplacian and Young's inequality to obtain a quantitative version of an existence theorem for non-constant Lipschitz harmonic functions on all infinite groups.

Statement (ii), which we discuss in section 7, is the heart of Kleiner's approach (inspired by the related work of Colding-Minicozzi $[\mathrm{CM}]$ ). His arguments are already quite quantitative. Two noticeable (related) difficulties that arise when trying to quantify Kleiner's proof are that the scale $R$ for which a finite-dimensional space of harmonic functions injects into the ball $B(e, R)$ is not effective, and that in this scale one lacks an a priori lower bound on the positive value of the determinant of an associated positive definite quadratic form.

For statement (iii), unlike the abstract setting of Kleiner's proof, the space we work with is the one appearing in statement (ii) (modulo the constants), in which the group operates naturally (preserving the Lipschitz norm). This is trivial in the qualitative world, but does require a certain amount of care in the quantitative setting (for instance, one needs quantitative versions of the assertion that any finitedimensional vector space has a basis), and is done in section 8 .

Statement (iv), established in section 9, was proven in [Kl] (as in Gromov's original $[\mathrm{Gr}]$ ) using the Tits alternative, or by a weaker variant of that alternative due to the first author [Sh]. However, for our purposes we provide a completely different, elementary proof, which makes crucial use of the fact (not exploited previously) that the linear representation in (iii) ranges in a compact Lie group (the one preserving the Lipschitz norm in (iii)). Our arguments here are inspired by the famous SolovayKitaev theorem [KiSV] in the theory of quantum computations, which itself may be viewed as a variant on the well-known Zassenhaus-Kazhdan-Margulis theorem (cf. $[\mathrm{K}, \S 4.12])$. As in the aforementioned results, we obtain a quantitative version of (iv) based on the fact that commutators of matrices near the identity collapse fast towards it, and hence cannot be non-trivially accommodated without a "rapidly growing supply" of group elements, which will be incompatible with the polynomial growth hypotheses. Furthermore, the compactness of the ambient group ensures the existence of a finite-index subgroup of matrices that are close enough to the identity that the previous considerations apply.

Statement (v) is formalized in Proposition 5.2, and follows simply by putting all the previous statements together.

Statements (vi), (vii) are formalized together as Proposition 5.3. Statement (vii) is a result of Milnor [Mi1] and Wolf [W]. The arguments of Milnor quickly allow us to reduce to a polycyclic setting in which $G$ is an extension of a cyclic group by 
a virtually nilpotent group. The main task, as in $[\mathrm{W}]$, is then to show that outer automorphisms of virtually nilpotent groups which are of polynomial growth are necessarily virtually unipotent. To do this, one must first eliminate the torsion from the nilpotent group, and then after taking quotients one is faced with understanding linear transformations of polynomial growth on a free abelian group $\mathbf{Z}^{d}$. But this can be handled using known results on Mahler measure of algebraic integers. It turns out that all these steps can be made semi-quantitative (in the sense that one does not control the index behind the modifier "virtually"), and this is done in sections 11-14; this then gives a quantitative (but ineffective) proof of Theorem 1.8 by a compactness argument given in section 3 . In section 15 we discuss the changes needed to make the semi-quantitative proof fully effective.

There are quite a few applications of Gromov's theorem where an effective version of it is of interest. We illustrate this only briefly in one geometric setting which was discussed in Gromov's original paper [Gr] as Corollary 15.2 below.

Finally, as a by-product of our effort one obtains a simplified, particularly accessible proof of Gromov's original qualitative theorem. It also avoids the Tits alternative and any use of $p$-adic numbers, and can be fully digested using basic background in linear algebra and calculus. This soft proof is presented in our companion paper [ShT], along with various other results and questions related to the current work and around the theme of Lipschitz harmonic functions on groups.

1.14 Comparison with other work. In the literature there seems to be only one previous effective result related to Gromov's theorem, due to van den Dries and Wilkie [DrW2], which handles the subquadratic growth case $d<2$ by using clever, elementary, and quite combinatorial arguments. (The case $d<1$ is trivial; see Lemma 5.1.)

A related quantitative formulation of Kleiner's argument was recently given by Lee and Makarychev [LeM]. They worked in the setting of a finite group $(G, S)$ obeying a doubling condition $\left|B_{S}(2 R)\right| \leq 2^{d}\left|B_{S}(R)\right|$ at all scales $R>0$, as opposed to the (weaker) assertion of being an $\left(R_{0}, d\right)$-growth group at a single scale. Using Kleiner's method, they obtained upper bounds (roughly of the order of $\exp \left(O\left(d^{2}\right)\right)$ ) on the multiplicity of eigenvalues of the Laplacian, and also obtained a subgroup of $G$ of index bounded by $\exp \left(\exp \left(O\left(d^{2}\right)\right)\right)$ which had a homomorphic image onto a cyclic group $\mathbf{Z} / M \mathbf{Z}$ of cardinality at least $M \gg \exp (-O(d))|G|^{\exp \left(-O\left(d^{2}\right)\right)}$. In the infinitary setting, the presence of a bounded index subgroup with a large homomorphic image can be used to locate a commutator subgroup with a reduced order of growth, to which one can apply an induction hypothesis to obtain a Gromov-like theorem. Unfortunately, a technical obstruction in this finitary case is that the doubling condition in [LeM] is required at all scales (up to the diameter of $G$ ), whereas the homomorphic image only yields a growth reduction up to scale $M$ or so. Our main Theorem 1.8 can be viewed as an answer to a question raised in [LeM], regarding whether Kleiner's methods can be adapted to the finitary setting assuming a polynomial growth hypothesis rather than a doubling condition. 
Another related result, of Milnor-Wolf type, was obtained recently by the second author in [T2]. There, it was shown that if $G$ is a $\left(R_{0}, d\right)$-growth group which is solvable of derived length at most $l$, with $R_{0}$ sufficiently large depending on $l, d$, then $G$ contains a nilpotent group of step at most $s(l, d)$ and index at most $R_{0}^{C(l, d)}$ for some $s(l, d), C(l, d)$ depending only on $l$ and $d$. This result has a much better control on the index of the nilpotent subgroup than Theorem 1.8, but it gives a much poorer (though still effective) bound on the step, or on the size of $R_{0}$ required, and is, in addition, restricted to the solvable case. The methods used in that paper are quite different from those here, replying on additive combinatorics rather than the theory of harmonic functions and linear representations. By combining the results in [T2] with Theorem 15.3, one can obtain a variant of Theorem 1.8 in which the bound $K\left(R_{0}, d\right)$ is completely effective, but the quantities $\exp \left(\exp \left(C d^{C}\right)\right)$ and $C^{d}$ appearing in that theorem are replaced by much larger (but still effective and explicit) functions of $d$. We omit the details.

1.15 Acknowledgments. The authors thank Emmanuel Breuillard for valuable discussions, and the anonymous referee for corrections. The first author was supported by ISF and NSF grants number 500/05 and DMS-0701639 resp. The second author is supported by a grant from the MacArthur Foundation, by NSF grant DMS-0649473, and by the NSF Waterman award.

\section{Notation}

If $E, F$ are two subsets of a multiplicative group $G$, we use $E \cdot F$ to denote the product set $\{e f: e \in E, f \in F\}$, and $E^{-1}$ to denote the inverse set $\left\{e^{-1}: e \in E\right\}$. In an additive group we can similarly define the sum set $E+F$ and reflection $-E$. We also define dilates $k \cdot E:=\{k e: e \in E\}$ for $E$ in an additive group and non-negative integers $k$.

Given a group $G$, we define the commutator $[g, h]$ of two group elements $g, h \in G$ by $[g, h]:=g h g^{-1} h^{-1}$, and the commutator of two subgroups $[H, K]$ to be the group generated by $\{[h, k]: h \in H, k \in K\}$. We define the lower central series $G=G_{1} \geq G_{2} \geq \ldots$ by $G_{1}:=G$ and $G_{i+1}:=\left[G_{i}, G\right]$, and the derived series $G=G^{(1)} \geq G^{(2)} \geq \ldots$ by $G^{(1)}:=G$ and $G^{(i+1)}:=\left[G^{(i)}, G^{(i)}\right]$. We say that $G$ is nilpotent of step at most $s$ if $G_{s+1}$ is trivial, and solvable of derived length at most $l$ if $G^{(l+1)}$ is trivial.

We write $X=O(Y), X \ll Y$ or $Y \gg X$ to denote the statement that $|X| \leq C Y$ for some absolute constant $C$.

\section{A Compactness Reduction}

In this section we perform a compactness reduction to eliminate the role of the quantities $K\left(R_{0}, d\right)$ appearing in Theorem 1.8. This will simplify the proof substantially, at the cost of rendering the final value of $K\left(R_{0}, d\right)$ obtained ineffective; but see section 15 for how one could avoid the use of compactness to obtain an effective value of $K\left(R_{0}, d\right)$. 
We first remove $K$ and $R$ from the definition of virtual nilpotency.

Definition 3.1 (Virtual nilpotency, again). Let $s, D \geq 1$. A finitely generated group $(G, S)$ is said to be virtually $(s, D)$-nilpotent if it contains a finite-index subgroup that is $(s, D)$-nilpotent.

We now claim that Theorem 1.8 follows from the "semi-quantitative" variant below:

Theorem 3.2 (Semi-quantitative Gromov theorem). Let $d>0$ and $R_{0}>0$, and assume that

$$
R_{0} \geq \exp \left(\exp \left(C d^{C}\right)\right)
$$

for some sufficiently large absolute constant $C$. Then every $\left(R_{0}, d\right)$-growth group is virtually $\left(C^{d}, C^{d}\right)$-nilpotent.

We now give the (standard) compactness argument that lets us deduce Theorem 1.8 from Theorem 3.2 (such an argument already appeared in Gromov's [Gr], while the general formalism of the "space of marked finitely generated groups" underlying it, was introduced later by Grigorchuk in [G2]).

Proof of Theorem 1.8 assuming Theorem 3.2. Let $C$ be the absolute constant in Theorem 3.2. Suppose Theorem 1.8 failed, then we could find $R_{0}, d$ obeying the specified bound (for this choice of $C$ ), and a sequence $\left(G_{N}, S_{N}\right)$ of $\left(R_{0}, d\right)$-growth groups with $N \rightarrow \infty$ such that $\left(G_{N}, S_{N}\right)$ is not $\left(N, N, C^{d}, C^{d}\right)$-virtually nilpotent. Observe that $\left|S_{N}\right| \leq\left|B_{S_{N}}\left(R_{0}\right)\right| \leq R_{0}^{d}$ is uniformly bounded, hence by passing to a subsequence we may assume $\left|S_{N}\right|=k$ for all $N$ and some fixed $k$. Now identify each $\left(G_{N}, S_{N}\right)$ as a quotient of the free group $\left(F_{k}, S\right)$ under the homomorphism mapping the free generators $S$ to $S_{N}$, and denote the kernel by $M_{N}<F_{k}$. By a diagonalization process and after passing to a subsequence, we may assume that the sets $M_{N}$ converge, i.e. they eventually agree on every finite subset of $F_{k}$. Their limit, $M$, is of course a normal subgroup of $F_{k}$. Obviously $G=F_{k} / M$ with the projection of $S$ is a $\left(R_{0}, d\right)$-growth group, hence by Theorem 3.2 it has a finite-index $\left(C^{d}, C^{d}\right)$-nilpotent subgroup. By Remark $1.4,(G, S)$ is a $\left(K, K, C^{d}, C^{d}\right)$-subgroup for some $K$. To complete the argument it only remains to observe the following three facts:

(1) The group $G$, being virtually nilpotent, is finitely presented;

(2) If a sequence of marked groups converges to a finitely presented group as above, then from some point on they are all quotients of it (indeed, this happens when the finitely many relations of the limit group stabilize in the sequence); and

(3) The property of being a $\left(K, K, C^{d}, C^{d}\right)$-nilpotent group is inherited by quotients.

For technical reasons it is convenient to modify the above definition slightly, by replacing the number $D$ of generators and the step $s$ with the Hirsch length of the nilpotent group. Recall that the Hirsch length of a nilpotent group (or more generally, a polycyclic group) is the sum of the torsion-free ranks of the quotients in 
any normal series of that group with abelian quotients (e.g. for a nilpotent group, one could use the lower central series).

Definition 3.3 (Virtual nilpotency, yet again). Let $r \geq 1$. A $r$-nilpotent group is a finitely generated nilpotent group of Hirsch length at most $r$ (and thus step at most $r$, as well). A finitely generated group $(G, S)$ is said to be virtually $r$-nilpotent if it contains a finite-index subgroup that is $r$-nilpotent.

By a result of Malcev [M2], if $G$ is nilpotent then any set $S \subset G$, whose projection generates the abelianization $G /[G, G]$, generates all of $G$. Hence, a $r$-nilpotent group contains a finite-index subgroup generated by at most $r$ generators (or $2 r$, if one enforces symmetry). Thus one may replace the conclusion of Theorem 3.2 by the assertion that every $\left(R_{0}, d\right)$-growth group is virtually $C^{d}$-nilpotent.

It remains to prove Theorem 3.2 with this modification. This is the purpose of the remaining sections of the paper.

\section{Generator Bounds}

In this section we collect a number of useful results which exploit polynomial growth hypotheses to locate bounded sets of generators for various types of groups; such results will be used frequently in the sequel.

First, we observe that the property of having quantitative finite index is transitive.

LEMma 4.1 (Transitivity of quantitative finite index). If $\left(G^{\prime}, S^{\prime}\right)$ is a $(K, R)$ subgroup of $(G, S)$, and $\left(G^{\prime \prime}, S^{\prime \prime}\right)$ is a $\left(K^{\prime}, R^{\prime}\right)$-subgroup of $\left(G^{\prime}, S^{\prime}\right)$, then $\left(G^{\prime \prime}, S^{\prime \prime}\right)$ is a $\left(K K^{\prime}\left(K+R K^{\prime}+1\right), R R^{\prime}\right)$-subgroup of $(G, S)$.

Proof. Clearly $S^{\prime \prime} \subset B_{S^{\prime}}\left(R^{\prime}\right) \subset B_{S}\left(R R^{\prime}\right)$. Next, since

$$
B_{S}(K+1) \subset B_{S}(K) \cdot B_{S^{\prime}}(K),
$$

we have

$$
B_{S}(n) \subset B_{S}(K+n) \subset B_{S}(K) \cdot B_{S^{\prime}}(n K)
$$

for any $n \geq 1$. Similarly

$$
B_{S}\left(n^{\prime}\right) \subset B_{S^{\prime}}\left(K^{\prime}+n^{\prime}\right) \subset B_{S^{\prime}}\left(K^{\prime}\right) \cdot B_{S^{\prime \prime}}\left(n^{\prime} K^{\prime}\right)
$$

for all $n^{\prime} \geq 1$. Combining the two, we conclude that

$$
B_{S}(n) \subset B_{S}(K) \cdot B_{S^{\prime}}\left(K^{\prime}\right) \cdot B_{S^{\prime \prime}}\left(n K K^{\prime}\right)
$$

for all $n \geq 1$. Since $B_{S^{\prime}}\left(K^{\prime}\right) \subset B_{S}\left(R K^{\prime}\right)$, we conclude that

$$
B_{S}(n) \subset B_{S}\left(K+R K^{\prime}\right) \cdot B_{S^{\prime \prime}}\left(n K K^{\prime}\right) .
$$

Setting $n:=K+R K^{\prime}+1$, we conclude that

$$
B_{S}\left(K+R K^{\prime}+1\right) \subset B_{S}\left(K+R K^{\prime}\right) \cdot B_{S^{\prime \prime}}\left(K K^{\prime}\left(K+R K^{\prime}+1\right)\right) .
$$

and thus

$$
B_{S}\left(K K^{\prime}\left(K+R K^{\prime}+1\right)+1\right) \subset B_{S}\left(K K^{\prime}\left(K+R K^{\prime}+1\right)\right) \cdot B_{S^{\prime \prime}}\left(K K^{\prime}\left(K+R K^{\prime}+1\right)\right)
$$

and the claim follows. 
Next, we observe that a $\left(R_{0}, d\right)$ group has a bounded number of generators, after passing to a finite-index subgroup.

LEMma 4.2 (Generator reduction of $\left(R_{0}, d\right)$-growth groups). Let $d \geq 1,0<\kappa<1$, and $R_{0} \geq 100^{1 / \kappa}$, and let $(G, S)$ be a $\left(R_{0}, d\right)$-growth group. Then there exists a $\left(R_{0}^{\kappa}, R_{0}^{\kappa}\right)$-subgroup $\left(G^{\prime}, S^{\prime}\right)$ of $G$ with $\left|S^{\prime}\right| \ll O(1)^{d / \kappa}$. In particular (by Remark 1.6) $\left(G^{\prime}, S^{\prime}\right)$ is a $\left(R_{0}^{1-\kappa}, d /(1-\kappa)\right)$-growth group. Furthermore we have $G=G^{\prime} \cdot B_{S}\left(R_{0}^{\kappa}\right)$.

REMARK 4.3. In practice, we will take $\kappa$ to be small compared to $d$ (e.g. $\kappa=1 / 100 d$ ), and so the slight degradation $d \mapsto d /(1-\kappa)$ in the order of growth here will be acceptable (at a later stage of the argument, we will obtain a reduction in the growth order by 1, which will more than compensate for these sorts of losses).

Proof. Clearly

$$
1 \leq\left|B_{S}(1)\right| \leq\left|B_{S}\left(R_{0}^{\kappa}\right)\right| \leq\left|B_{S}\left(R_{0}\right)\right| \leq R_{0}^{d} .
$$

By the pigeonhole principle, one can thus find a radius $1 \leq r \leq R_{0}^{\kappa} / 10$ such that

$$
\left|B_{S}(10 r)\right| \ll O(1)^{d / \kappa}\left|B_{S}(r)\right| \text {. }
$$

Fix this $r$. Now, let $X$ be a maximal subset of $B_{S}(4 r)$ such that the sets $x \cdot B_{S}(r)$ for $x \in X$ are disjoint. Since the $x \cdot B_{S}(r)$ are contained in $B_{S}(5 r)$, we have

$$
|X| \leq\left|B_{S}(5 r)\right| /\left|B_{S}(r)\right| \ll O(1)^{d / \kappa} \text {. }
$$

On the other hand, by construction of $X$ we have the covering property

$$
B_{S}(4 r) \subset X \cdot B_{S}(2 r) \text {. }
$$

In particular, if we set $S^{\prime}:=X \cup X^{-1}$, and let $G^{\prime}$ be the group generated by $S^{\prime}$, then $\left|S^{\prime}\right| \ll O(1)^{d / \kappa}$ and

$$
\begin{gathered}
B_{S}(4 r) \subset S^{\prime} \cdot B_{S}(2 r), \\
B_{S}(n r) \subset B_{S^{\prime}}(n) \cdot B_{S}(2 r),
\end{gathered}
$$

for $n=1,2, \ldots$. In particular, we have

$$
B_{S}\left(R_{0}^{\kappa}+1\right) \subset B_{S^{\prime}}\left(R_{0}^{\kappa}\right) \cdot B_{S}\left(R_{0}^{\kappa}\right)
$$

which on inversion gives

$$
B_{S}\left(R_{0}^{\kappa}+1\right) \subset B_{S}\left(R_{0}^{\kappa}\right) \cdot B_{S^{\prime}}\left(R_{0}^{\kappa}\right) .
$$

On the other hand, we have $S^{\prime} \subset B_{S}(4 r) \subset B_{S}\left(R_{0}^{\kappa}\right)$, and the claim follows.

REMARK 4.4. Because of this lemma, we will be able to safely absorb a number of terms involving the size $|S|$ of the generating set in the arguments that follow. (One should think of $d$ and $1 / \kappa$ as being bounded; the key point is that the bound on $|S|$ is independent of $R_{0}$.)

REMARK 4.5. If one does not pass to a finite-index subgroup, then one may need as many as $\log R_{0}$ generators. Indeed, consider the abelian group $G=\mathbf{Z}_{2}^{n} \times \mathbf{Z}$, where $n \sim \log R_{0}$. Then $B_{S}\left(R_{0}\right)$ is of polynomial size in $R_{0}$, but one needs $n+1 \sim \log R_{0}$ generators in order to generate the whole group $G$. In the converse direction, if $B_{S}\left(R_{0}\right) \leq R_{0}^{d}<2^{R_{0}}$, then if we let $s_{1}, \ldots, s_{n}$ be a maximal sequence in $S$ which is 
dissociated (i.e. the words $s_{1}^{i_{1}} \ldots s_{n}^{i_{n}}$ for $i_{1}, \ldots, i_{n} \in\{0,1\}$ are different), then one easily verifies that $2^{n} \leq\left|B_{S}\left(R_{0}\right)\right| \leq R_{0}^{d}<2^{R_{0}}$ and thus $n \leq d \log _{2} R_{0}<R_{0}$, and that the set $\left\{s_{1}, \ldots, s_{n}, s_{1}^{-1}, \ldots, s_{n}^{-1}\right\}$ generates $S$ and thus $G$. Thus $G$ can be generated by $O\left(d \log R_{0}\right)$ generators in this case.

REMARK 4.6. In many applications of Theorem 1.8, $S$ would already be bounded. But in our proof of Theorem 1.8 (which is based on an induction on $d$ ), it will become necessary at some stage in the proof to pass from $G$ to a subgroup such as $[G, G]$, which need not have a bounded number of generators. It is then that Lemma 4.2 becomes necessary.

We shall need two further results in a similar spirit. The first asserts that if a $\left(R_{0}, d\right)$-growth group $G$ can be generated by a small number of generators and has polynomial growth, then so does $[G, G]$ (cf. [Gr, p.61], or [Mi1]):

LEMma 4.7 (Generator reduction for a commutator group). Let $d \geq 1,0<\kappa<1$, and $R_{0} \geq C d^{C / \kappa}$ for some sufficiently large absolute constant $C$, and let $(G, S)$ be a $\left(R_{0}, d\right)$-growth group. Then there exists a set of generators $S^{\prime}$ of $[G, G]$, each of the form $g\left[e, e^{\prime}\right] g^{-1}$ for some $e, e^{\prime} \in S$ and $g \in B_{S}\left(\frac{1}{2} R_{0}^{\kappa}-4\right)$. In particular, $\left([G, G], S^{\prime}\right)$ is a $\left(\infty, R_{0}^{\kappa}\right)$-subgroup of $(G, S)$, and thus (by Remark 1.6) $\left([G, G], S^{\prime}\right)$ is a $\left(R_{0}^{1-\kappa}, d /(1-\kappa)\right)$-growth group.

Proof. It is not difficult to see that $[G, G]$ is generated by the elements $g\left[e, e^{\prime}\right] g^{-1}=$ $\left[g e g^{-1}, g e^{\prime} g^{-1}\right]$ where $e, e^{\prime} \in S$ and $g \in G$ (since modulo the normal group generated by these elements, all the basis elements $e, e^{\prime}$ commute). The difficulty is to replace this infinite generating set by a finite one.

For any $r \geq 1$, let $A_{r}:=\left\{g\left[e, e^{\prime}\right] g^{-1}: e, e^{\prime} \in S, g \in B_{S}(r)\right\} \subset B_{S}(2 r+4)$, and let $A_{\leq r}:=A_{r} \cdot A_{r-1} \ldots \cdot A_{0}$. Then $A_{\leq r} \subset B_{S}\left(O(r+1)^{2}\right)$, and thus by hypothesis we have $\left|A_{\leq r}\right| \leq R_{0}^{d}$ for $r \leq c R_{0}^{1 / 2}$ and some small absolute constant $c>0$. By hypothesis (and if $C$ is large enough), $R_{0}^{\kappa / 2}$ is larger than a large multiple of $d \log R_{0}$. In particular, one can find a $2 \leq r_{0} \leq \frac{1}{2} R_{0}^{\kappa}-4$ such that

$$
\left|A_{\leq r_{0}}\right|<2^{r_{0}-2} \text {. }
$$

From the pigeonhole principle, we can thus find $2 \leq r \leq r_{0}$ such that

$$
\left|A_{\leq r+1}\right|<2\left|A_{\leq r}\right| .
$$

This implies that for any $x \in A_{r+1}$, that $x \cdot A_{\leq r}$ and $A_{\leq r}$ overlap, thus

$$
A_{\leq r+1} \subset A_{\leq r} \cdot A_{\leq r}^{-1} .
$$

In particular, $A_{r+1}$ is contained in the group generated by $A_{r}$, which implies that the act of conjugation by any element $g \in S$ preserves the group generated by $A_{r}$. Since $A_{r}$ also contains the commutators $\left[e, e^{\prime}\right]$ for $e, e^{\prime} \in S$, we conclude that $S^{\prime}:=A_{r}$ generates $[G, G]$; also, $S^{\prime}$ is symmetric by construction. The claims of the lemma then follow.

Next, we show that finite-index subgroups of finitely generated groups continue to be finitely generated in a very quantitative manner. 
LEMmA 4.8 (Qualitative finite index implies quantitative finite index). Let $d \geq 1$, $I \geq 1$. Let $G=(G, S)$ be a finitely generated group, and let $G^{\prime}$ be a finite-index subgroup of $G$ with index $\left|G: G^{\prime}\right| \leq I$. Then there exists a set $S^{\prime} \subset B_{S}(2 I+1)$ of generators of $G^{\prime}$ such that $\left(G^{\prime}, S^{\prime}\right)$ is a $(2 I+1,2 I+1)$-subgroup of $G$.

Proof. For each $r>0$, the set $B_{S}(r) \cdot G^{\prime}$ is a union of left cosets of $G^{\prime}$. The number of such cosets is of course $\left|G: G^{\prime}\right|$. Thus by the pigeonhole principle, one can find $0 \leq r_{0} \leq I$ such that $B_{S}\left(r_{0}+1\right) \cdot G^{\prime}=B_{S}\left(r_{0}\right) \cdot G^{\prime}$. In particular,

$$
B_{S}\left(r_{0}+1\right) \subset B_{S}\left(r_{0}\right) \cdot G^{\prime} \text {. }
$$

If we set $S^{\prime}:=G^{\prime} \cap B_{S}\left(2 r_{0}+1\right)$, then $S^{\prime}$ is symmetric and contained in $B_{S}(2 I+1)$, and by the triangle inequality we have

$$
B_{S}\left(r_{0}+1\right) \subset B_{S}\left(r_{0}\right) \cdot S^{\prime}
$$

Multiplying both sides on the left by $B_{S}\left(r-r_{0}\right)$, we obtain

$$
B_{S}(r+1) \subset B_{S}(r) \cdot S^{\prime}
$$

for all $r \geq r_{0}$ (and hence for all $r \geq 2 I+1$ ). In particular

$$
B_{S}(2 I+2) \subset B_{S}(2 I+1) \cdot B_{S^{\prime}}(2 I+1)
$$

and the claim follows.

\section{Reduction to Two Key Propositions}

Theorem 3.2 (modified as discussed at the end of section 3) is deduced from one easy proposition and two difficult ones. We begin with the easy proposition, which handles the base case $d<1$ :

Lemma 5.1( Sublinear growth case). Suppose that $(G, S)$ is a $(R, d)$-growth group for some $0<d<1$ and $R>1$. Then $G=B_{S}(R)$ and so $G$ is finite with $|G| \leq R^{d}$.

Proof. The $\left|B_{S}(r)\right|$ for $0 \leq r<R$ are integers between 1 and $\left|B_{S}(R)\right|=R^{d}<R$ that increase in $r$. Thus by the pigeonhole principle, there exists $0 \leq r<R-1$ such that $\left|B_{S}(r)\right|=\left|B_{S}(r+1)\right|$, thus $B_{S}(r)=B_{S}(r+1)$. Iterating this we see that $B_{S}(r)=B_{S}\left(r^{\prime}\right)$ for all $r^{\prime} \geq r$, thus $G=B_{S}(r)=B_{S}(R)$ and the claim follows.

The higher-order growth cases $d \geq 1$ will then be handled by an induction on $d$. The first major step in this process is to obtain a reduction in the order of growth for a certain commutator subgroup:

Proposition 5.2 (Reduction in growth order). Let $R_{0}, d \geq 1$, and let $(G, S)$ be a $\left(R_{0}, d\right)$-growth group. Assume that

$$
R_{0} \geq \exp \left(\exp \left(C d^{C}\right)\right)
$$

for some sufficiently large absolute constant $C$. Then at least one of the following holds:

(i) $G=B_{S}\left(R_{0}^{\exp \left(\exp \left(O(d)^{O(1)}\right)\right)}\right)$ (in particular, $G$ is finite). 
(ii) There exists a $\left(R_{0}^{1 / 10}, R_{0}^{1 / 10}\right)$-subgroup $\left(G^{\prime}, S^{\prime}\right)$ of $G$ and a positive integer $l=$ $O\left(d^{O(1)}\right)$ such that $\left(G^{\prime}\right)^{(l)}$ is generated by a set $\left(S^{\prime}\right)^{(l)} \subset B_{S^{\prime}}\left(R_{0}^{1 / 10}\right)$ for which $\left(\left(G^{\prime}\right)^{(l)},\left(S^{\prime}\right)^{(l)}\right)$ is a $(R, d-0.9)$-growth group for some $R_{0}^{1-1 / 10 d} \leq R \leq R_{0}$.

This proposition will be established in sections 6-10 using (quantitative versions of) some arguments of Kleiner [Kl]. The bounds here are completely effective (and far superior to those in Proposition 5.3 below).

To exploit this growth reduction we need the following variant of Theorem 1.8.

Proposition 5.3 (Semi-quantitative Milnor-Wolf theorem). Let $s, l, r, R_{0}, d \geq 1$, and suppose that

$$
R_{0} \geq r^{C}(C d)^{C d l}
$$

for some sufficiently large absolute constant $C$. Suppose we have a short exact sequence

$$
0 \rightarrow H \rightarrow G \rightarrow L \rightarrow 0
$$

of groups, where $G=(G, S)$ is a $\left(R_{0}, d\right)$-growth group, $H=\left(H, S_{H}\right)$ is a virtually $r$ nilpotent (see Definition 3.3) $\left(\infty, R_{0}^{1 / 10}\right)$-subgroup of $G$, and $L$ is solvable of derived length at most $l$. Then $(G, S)$ is virtually $r+l O(1)^{d}$-nilpotent.

We prove this proposition in sections 11-14. The arguments are quite different from those used to prove Proposition 5.2, being related to the arguments used by Milnor [Mi1] and Wolf [W]. A key new technical difficulty in this "single-scale" setting, not present in earlier "multi-scale" work, is that the index of the nilpotent subgroup of $H$ may be so large that this subgroup is not "visible" at the one scale $R_{0}$ that we directly control.

In the remainder of this section we show how the above three propositions imply Theorem 3.2 (modified as discussed at the end of section 3). From Lemma 5.1 we see that Theorem 3.2 holds for $d<1$. We may thus assume inductively that $d \geq 1$ and that the claim has already been proven for $d-0.9$. By Proposition 5.2, we see that either conclusion (i) or conclusion (ii) of that proposition holds. If conclusion (i) holds, then $G$ is finite and the claim follows. If instead conclusion (ii) holds, then by induction hypothesis we see that $\left(\left(G^{\prime}\right)^{(l)},\left(S^{\prime}\right)^{(l)}\right)$ is virtually $C^{d-0.9}$-nilpotent. On the other hand, $G^{\prime} /\left(G^{\prime}\right)^{(l)}$ is clearly solvable of derived length at most $l=O\left(d^{O(1)}\right)$. Applying Proposition 5.3 to the short exact sequence

$$
0 \rightarrow\left(G^{\prime}\right)^{(l)} \rightarrow G^{\prime} \rightarrow G^{\prime} /\left(G^{\prime}\right)^{(l)} \rightarrow 0
$$

we conclude (if $C$ is large enough) that $G^{\prime}$ is virtually $C^{d}$-virtually nilpotent. Since $G^{\prime}$ is a finite-index subgroup of $G$, the claim then follows.

\section{First Step for Proposition 5.2: Production of a Non-Trivial Lipschitz Almost Harmonic Function}

We now begin the proof of Proposition 5.2, following Kleiner [Kl]. Kleiner's argument is based on harmonic functions, and in particular on studying the class of functions $u: G \rightarrow \mathbf{R}$ which are both Lipschitz and harmonic with respect to the 
set $S$ of generators. In our quantitative applications, the group $G$ could well be finite (with extremely large cardinality), and so there may not be any harmonic functions other than the constants. To deal with this we need to consider instead a somewhat larger class of almost harmonic functions. A good example is provided on the finite group $G=\mathbf{Z} / N \mathbf{Z}$ (with the standard generators $+1,-1$, and with $N$ large) by the function $u(x):=N \sin (2 \pi x / N)$. This function is Lipschitz (with Lipschitz norm approximately $2 \pi$ ), and "almost harmonic" in the sense that $u(x+1)+u(x-1)-2 u(x)=O(1 / N)$ for all $x$.

It is known (see [Kl, App.] and the references therein) that infinite groups admit non-trivial Lipschitz harmonic functions. It is thus not surprising that all "large" groups (finite or infinite) admit non-trivial almost harmonic Lipschitz functions. To see this, let us first formalize our definitions.

Definition 6.1. Let $u: G \rightarrow \mathbf{R}$ be a function. The gradient $\nabla u: G \rightarrow \mathbf{R}^{S}$ of $u$ is defined by the formula

$$
\nabla u(x):=(u(x s)-u(x))_{s \in S},
$$

so in particular

$$
|\nabla u(x)|:=\left(\sum_{s \in S}|u(x s)-u(x)|^{2}\right)^{1 / 2} .
$$

Dually, given a vector-valued function $F=\left(F_{s}\right)_{s \in S}: G \rightarrow \mathbf{R}^{S}$, we can define its divergence $\nabla \cdot F: G \rightarrow \mathbf{R}$ by the formula

$$
\nabla \cdot\left(F_{s}\right)_{s \in S}(x)=\sum_{s \in S} F_{s}\left(x s^{-1}\right)-F_{s}(x) .
$$

The Laplacian of a function $u: G \rightarrow \mathbf{R}$ is defined by the formula

$$
\Delta u:=-\nabla \cdot \nabla u
$$

or more explicitly (using the symmetry of $S$ )

$$
\Delta u(x)=2|S| u(x)-2 \sum_{s \in S} u(x s) .
$$

The Lipschitz (semi)-norm $\|u\|_{\text {Lip }}$ of $u$ is defined as

$$
\|u\|_{\text {Lip }}:=\|\nabla u\|_{\ell^{\infty}(G)}=\sup _{x \in G}|\nabla u(x)| .
$$

A function $u: G \rightarrow \mathbf{R}$ is harmonic if $\Delta u=0$. If $\varepsilon>0$, we say that a function $u: G \rightarrow \mathbf{R}$ is $\varepsilon$-harmonic Lipschitz if $\|u\|_{\text {Lip }} \leq 1$ and $\|\Delta u\|_{\ell^{\infty}(G)} \leq \varepsilon$.

REMARK 6.2. The conventions for the Laplacian $\Delta$ and Lipschitz norm may differ by some (ultimately irrelevant) constant factors from other definitions in the literature. With these conventions, the function $\frac{1}{2 \pi} N \sin (2 \pi x / N)$ is $O(1 / N)$-harmonic Lipschitz on $\mathbf{Z} / N \mathbf{Z}$.

Proposition 6.3 (Infinite groups have non-constant Lipschitz harmonic functions). Let $R \geq 1$, and let $(G, S)$ be a finitely generated group. Then at least one of the following statements is true: 
- $G=B_{S}(R)$.

- There exists a $O\left(|S| R^{-1 / 3}\right)$-harmonic Lipschitz function $u: G \rightarrow \mathbf{R}$ with $|\nabla u(\mathrm{id})| \geq 1 /|S|$.

Remark 6.4. Comparing this with the example $u(x)=\frac{1}{2 \pi} N \sin (2 \pi x / N)$ on $\mathbf{Z} / N \mathbf{Z}$ we see that the exponent $-1 / 3$ in the proposition should probably be a -1 . However, any positive exponent will suffice for our application.

Proof. Given two functions $u, v: G \rightarrow \mathbf{R}$, we formally define the convolution $u * v$ : $G \rightarrow \mathbf{R}$ by the formula

$$
u * v(x):=\sum_{y \in G} u(y) v\left(y^{-1} x\right)=\sum_{y \in G} u\left(x y^{-1}\right) v(y) .
$$

By Young's inequality, convolution is well-defined whenever $u \in \ell^{p}(G), v \in \ell^{q}(G)$ and $1 / p+1 / q=1 / r+1$ for some $1 \leq p, q, r \leq \infty$, in which case we have

$$
\|u * v\|_{\ell^{r}(G)} \leq\|u\|_{\ell^{p}(G)}\|v\|_{\ell^{q}(G)} .
$$

We observe the pleasant identities

$$
\begin{gathered}
\nabla\left(f_{1} * f_{2}\right)=f_{1} * \nabla f_{2}, \\
\nabla \cdot(f * F)=f *(\nabla \cdot F),
\end{gathered}
$$

and thus

$$
\Delta\left(f_{1} * f_{2}\right)=f_{1} * \Delta f_{2} .
$$

Fix $R$. We may assume that $G \neq B_{S}(R)$, since we are done otherwise. Let $\sigma$ be the measure $\sigma=\frac{1}{|S|} \sum_{s \in S} \delta_{s}$, where $\delta_{s}$ is the Kronecker delta. Observe that $\Delta u=2|S|(u * \sigma-u)$ for any $u$, where $u * \sigma(x):=\sum_{y} u\left(x y^{-1}\right) \sigma(y)$ is the usual convolution operator.

Let $\sigma^{(m)}:=\sigma * \ldots * \sigma$ be the $m$-fold convolution of $\sigma$, and let $f: G \rightarrow \mathbf{R}$ be the function

$$
f:=\frac{1}{R} \sum_{m=0}^{R} \sigma^{(m)} .
$$

Then we have $\|f\|_{\ell^{1}(G)}=1$ and $\|\Delta f\|_{\ell^{1}(G)} \ll|S| / R$. We divide into two cases.

Case 1 ("non-amenable" case): $\|\nabla f\|_{\ell^{1}(G)} \geq R^{-2 / 3}$. By the pigeonhole principle, we can then find $s \in S$ such that the function $f_{s}(x):=f(x s)-f(x)$ has $\ell^{1}$ norm at least $|S|^{-1}\|\nabla f\|_{\ell^{1}(G)}$. If we then define $u:=h * f$, where $h(x):=$ $\operatorname{sgn}\left(f_{s}\left(x^{-1}\right)\right) /\|\nabla f\|_{\ell^{1}(G)}$, we see from (6), (7) that

$$
\|\nabla u\|_{\ell^{\infty}(G)} \leq 1
$$

and

$$
|u(s)-u(\mathrm{id})| \geq 1 /|S|,
$$

while from (6), (9) we have

$$
\|\Delta u\|_{\ell^{\infty}(G)} \ll(|S| / R) / R^{-2 / 3},
$$

and the claim follows. 
Case 2. ("amenable" case): $\|\nabla f\|_{l^{1}(G)}<R^{-2 / 3}$. If we set $F:=|f|^{1 / 2}$ then we have the pointwise bound

$$
|\nabla F| \leq|S|^{1 / 2}|\nabla f|^{1 / 2}
$$

thanks to the elementary estimate $|x|^{1 / 2}-|y|^{1 / 2} \leq|x-y|^{1 / 2}$ for $x, y \geq 0$. Thus we have

$$
\|F\|_{\ell^{2}(G)}=1 \quad \text { and } \quad\|\nabla F\|_{\ell^{2}(G)} \leq|S|^{1 / 2} R^{-1 / 3} .
$$

Also, $F$ is supported on $B_{S}(R)$. Since $G \neq B_{S}(R)$ by hypothesis, $F$ cannot be constant. In particular

$$
\|\nabla F\|_{\ell^{2}(G)}>0 \text {. }
$$

Thus if $\mu_{F}$ is the spectral measure of $\Delta$ relative to $F$, we see that $\mu_{F}$ is a probability measure on $[0,1]$ with

$$
0<\int_{0}^{1} x d \mu_{F}(x)=\|\nabla F\|_{\ell^{2}(G)}^{2} \leq|S| R^{-1 / 3} .
$$

This implies that

$$
0<\int_{0}^{|S| R^{-1 / 3}} x^{2} d \mu_{F}(x) \leq|S| R^{-1 / 3} \int_{0}^{|S| R^{-1 / 3}} x d \mu_{F}(x) .
$$

If we then let $F^{\prime} \in \ell^{2}(G)$ be the spectral projection of $F$ to $\left[0,|S| R^{-1 / 3}\right]$, we conclude that

$$
0<\left\|\Delta F^{\prime}\right\|_{\ell^{2}(G)}^{2} \leq|S| R^{-1 / 3}\left\|\nabla F^{\prime}\right\|_{\ell^{2}(G)}^{2} .
$$

By the pigeonhole principle, we can find $s \in S$ such that the function $F_{s}^{\prime}(x):=$ $F^{\prime}(x s)-F^{\prime}(x)$ obeys the lower bound

$$
\left\|F_{s}^{\prime}\right\|_{\ell^{2}(G)}^{2} \geq|S|^{-1}\left\|\nabla F^{\prime}\right\|_{\ell^{2}(G)}^{2} .
$$

If we define $u:=H * F$, where $H(x):=F_{s}^{\prime}\left(x^{-1}\right) /\|\nabla F\|_{\ell^{2}(G)}^{2}$, we see from (6), (8) that

$$
\|\nabla u\|_{\ell^{\infty}(G)} \leq 1
$$

while from (11) we have

$$
|u(s)-u(\mathrm{id})| \geq 1 /|S|
$$

and from (6), (9), (10) we have

$$
\|\Delta u\|_{\ell^{\infty}(G)} \leq|S| R^{-1 / 3},
$$

and the claim follows.

REMARK. See also the recent paper $[\mathrm{LeP}]$ for a related probabilistic construction of (Hilbert space valued) harmonic maps on amenable groups.

Once one has one non-trivial almost harmonic function $u$, one can then create a further family $\rho(g) u, g \in G$, of almost harmonic functions by translation: $\rho(g) u(x):=u\left(g^{-1} x\right)$. Kleiner's approach to Gromov's theorem revolves around a study of this family, starting with the fundamental observation that this family is (approximately) finite-dimensional. We now turn to this important fact. 


\section{Second Step for Proposition 5.2: Kleiner's Theorem}

Kleiner $[\mathrm{Kl}]$, building upon work of Colding and Minicozzi $[\mathrm{CM}]$, proved that in a group of polynomial growth the space of Lipschitz harmonic functions is finitedimensional; indeed, an inspection of the argument shows that the dimension of this space is bounded by some constant $C(d)$ depending only on the order $d$ of growth. The main objective of this section is to prove the following quantitative version of this fact:

Theorem 7.1 (Quantitative Kleiner theorem). There exists an absolute constant $C>0$ such that for any $0<\kappa<0.1$, and any $\left(R_{0}, d\right)$-growth group $(G, S)$ with $d \geq 1$ and

$$
R_{0} \geq K:=(C|S|)^{C d^{3} / \kappa^{2}},
$$

there exists a finite-dimensional subspace $V$ of $\mathbf{R}^{G}$ of dimension

$$
\operatorname{dim}(V)=O(|S|)^{O\left(d^{3} / \kappa^{2}\right)}
$$

such that for every $R_{0}^{-K}$-harmonic Lipschitz function $u: G \rightarrow \mathbf{R}$, there exists $v \in V$ such that

$$
\|u-v\|_{\ell^{2}\left(B_{S}\left(R_{0}^{1-\kappa}\right)\right)} \leq R_{0}^{-100 d} .
$$

We will prove Theorem 7.1 in several stages, following [Kl]. For any $x \in G$ and $r \geq 1$, we write $B(x, r):=x \cdot B_{S}(r)$ for the ball of radius $r$ centered at $x$. The first step is the following Poincaré inequality (compare with one first proved by Colding and Minicozzi in $[\mathrm{CM}]$, and its adaptation to the group setting due to Kleiner-Saloff-Coste in [Kl, Th. 2.2]):

Lemma 7.2 (Poincaré inequality). Let $f: G \rightarrow \mathbf{R}, x \in G$, and $r \geq 1$. Let $f_{B(x, r)}:=\frac{1}{|B(x, r)|} \int_{B(x, r)} f$ be the average value of $f$ on $B(x, r)$. Then

$$
\left\|f-f_{B(x, r)}\right\|_{\ell^{2}(B(x, r))} \leq 2 r \frac{\left|B_{S}(2 r)\right|}{\left|B_{S}(r)\right|}\|\nabla f\|_{\ell^{2}(B(x, 3 r))} .
$$

Proof. By definition of the gradient, we have the pointwise bound

$$
|f(y g s)-f(y g)| \leq|\nabla f(y g)|
$$

for all $y, g \in G$ and $s \in S$. If we take $g \in B_{S}(2 r)$ and average this in $\ell^{2}$ over all $y \in B(x, r)$, we conclude that

$$
\left(\sum_{y \in B(x, r)}|f(y g s)-f(y g)|^{2}\right)^{1 / 2} \leq\|\nabla f\|_{\ell^{2}(B(x, 3 r))} .
$$

Telescoping this using the triangle inequality, we conclude that

$$
\left(\sum_{y \in B(x, r)}|f(y g)-f(y)|^{2}\right)^{1 / 2} \leq 2 r\|\nabla f\|_{\ell^{2}(B(x, 3 r))} .
$$

for all $g \in B_{S}(2 r)$. Summing in $g$ using the triangle inequality, we conclude that

$$
\left(\sum_{y \in B(x, r)}\left(\sum_{g \in B_{S}(2 r)}|f(y g)-f(y)|\right)^{2}\right)^{1 / 2} \leq 2 r\left|B_{S}(2 r)\right|\|\nabla f\|_{\ell^{2}(B(x, 3 r))} .
$$


But for any $y \in B(x, r)$, we have

$$
\left|f(y)-f_{B(x, r)}(y)\right| \leq \frac{1}{\left|B_{S}(r)\right|} \sum_{z \in B(x, r)}|f(z)-f(y)| \leq \frac{1}{\left|B_{S}(r)\right|} \sum_{g \in B_{S}(2 r)}|f(y g)-f(y)|,
$$

and the claim follows.

For $\varepsilon$-harmonic functions, we have a reverse inequality.

Lemma 7.3 (Reverse Poincaré inequality). Let $f: G \rightarrow \mathbf{R}$ be $\varepsilon$-harmonic Lipschitz, and let $B(x, r)$ be a ball for some $r \geq 1$. Then

$$
\|\nabla f\|_{\ell^{2}(B(x, r))} \ll|S|^{O(1)}\left(\frac{1}{r}\|f\|_{\ell^{2}(B(x, 2 r))}+\varepsilon r\left|B_{S}(2 r)\right|^{1 / 2}\right) .
$$

Proof. Let $\psi: G \rightarrow \mathbf{R}$ be the cutoff function $\psi(y):=\max \left(1-\frac{\operatorname{dist}(x, y)}{2 r}, 0\right)$. Then it will suffice to show that

$$
\sum_{y \in G}|\nabla f|^{2} \psi^{2}(y) \ll|S|^{O(1)}\left(\frac{1}{r^{2}}\|f\|_{\ell^{2}(B(x, 2 r))}^{2}+\varepsilon^{2} r^{2}\left|B_{S}(2 r)\right|\right) .
$$

We may clearly restrict the sum on the left to $B(x, 2 r-1)$. Now, for any $y \in B(x, 2 r-1)$ and $s \in S$ we have

$$
\begin{aligned}
(f(y s)-f(y)) \psi^{2}(y)=\left(f \psi^{2}(y s)-f \psi^{2}(y)\right)-f(y s) \psi(y)(\psi(y s)-\psi(y)) & \\
& -f(y s)(\psi(y s)-\psi(y))^{2} .
\end{aligned}
$$

From the triangle inequality, $\operatorname{dist}(x, y s)$ differs from $\operatorname{dist}(x, y)$ by at most 1 , and thus

$$
\psi(y s)-\psi(y)=O(1 / r) \text {. }
$$

Also, $f(y s)=O(|f(y)|+|\nabla f(y)|)$. Multiplying (14) by $f(y s)-f(y)$ and summing in $s$, we conclude that

$$
|\nabla f|^{2} \psi^{2}(y)=\nabla\left(f \psi^{2}\right) \cdot \nabla f+O\left(|S|(|f(y)|+|\nabla f(y)|)\left(\frac{\psi(y)}{r}+\frac{1}{r^{2}}\right)\right) .
$$

Inserting this into the left-hand side of (13) and summing by parts, we conclude that

$$
\begin{array}{r}
\sum_{y \in G}|\nabla f|^{2} \psi^{2}(y) \ll \sum_{y \in G}\left|f \psi^{2}(y)\right||\Delta f(y)|+\frac{|S|}{r} \sum_{y \in G}|\nabla f(y)|(|f(y)|+|\nabla f(y)|) \psi(y) \\
+\frac{|S|}{r^{2}} \sum_{y \in B(x, 2 r-1)}|\nabla f(y)|(|f(y)|+|\nabla f(y)|) . \quad \text { (15) }
\end{array}
$$

As $f$ is $\varepsilon$-harmonic, we can use Cauchy-Schwarz to bound

$$
\begin{aligned}
\sum_{y \in G}\left|f \psi^{2}(y) \| \Delta f(y)\right| & \leq \varepsilon \sum_{y \in B_{S}(2 r-1)}|f(y)| \\
& \leq \varepsilon\left|B_{S}(2 r)\right|^{1 / 2}\|f\|_{\ell^{2}\left(B_{S}(x, 2 r-1)\right)} \\
& \leq \varepsilon^{2} r^{2}\left|B_{S}(2 r)\right|+\frac{1}{r^{2}}\|f\|_{\ell^{2}\left(B_{S}(x, 2 r-1)\right)}^{2} .
\end{aligned}
$$

Another application of Cauchy-Schwarz gives 


$$
\begin{aligned}
& \frac{|S|}{r} \sum_{y \in G}|\nabla f(y)|(|f(y)|+|\nabla f(y)|) \psi(y) \\
& \leq c \sum_{y \in G}|\nabla f(y)|^{2} \psi^{2}(y)+\frac{1}{c} \frac{|S|^{2}}{r^{2}} \sum_{y \in G}(|f(y)|+|\nabla f(y)|)^{2}
\end{aligned}
$$

for any $c>0$. By choosing $c$ small enough, we can then absorb the first term on the right-hand side into the left-hand side of (15) and conclude that

$$
\sum_{y \in G}|\nabla f|^{2} \psi^{2}(y) \ll \varepsilon^{2} r^{2}\left|B_{S}(2 r)\right|+\frac{|S|^{O(1)}}{r^{2}}\left(\|f\|_{\ell^{2}\left(B_{S}(x, 2 r-1)\right)}^{2}+\|\nabla f\|_{\ell^{2}\left(B_{S}(x, 2 r-1)\right)}^{2}\right) .
$$

But from definition of $\nabla$ and the triangle inequality we see that

$$
\|\nabla f\|_{\ell^{2}\left(B_{S}(x, 2 r-1)\right)} \ll|S|\|f\|_{\ell^{2}\left(B_{S}(x, 2 r)\right)}
$$

and the claim follows.

Now we need some more definitions. Given $R>0$, define the symmetric bilinear form $Q_{R}: \mathbf{R}^{G} \times \mathbf{R}^{G} \rightarrow \mathbf{R}$ by the formula

$$
Q_{R}(u, v):=\sum_{x \in B_{S}(R)}(u(x)-u(\mathrm{id}))(v(x)-v(\mathrm{id})) .
$$

This form is clearly positive semi-definite (with finite rank). Given any $u_{1}, \ldots, u_{k}$ : $G \rightarrow \mathbf{R}$, we define the $R$-volume $\operatorname{Vol}_{R}\left(u_{1}, \ldots, u_{k}\right)$ by the formula

$$
\operatorname{Vol}_{R}\left(u_{1}, \ldots, u_{k}\right)=\operatorname{det}\left(\left(Q_{R}\left(u_{i}, u_{j}\right)\right)_{1 \leq i, j \leq k}\right)^{1 / 2} ;
$$

the right-hand side is non-negative due to the positive semi-definite nature of $Q_{R}$. Geometrically, one can view $\operatorname{Vol}_{R}\left(u_{1}, \ldots, u_{k}\right)$ as the length of the wedge product $u_{1} \wedge \ldots \wedge u_{k}$ in $\wedge^{k} \mathbf{R}^{G}$, using the induced semi-definite form from $Q_{R}$.

The $Q_{R}$ are monotone increasing in $R$ as symmetric bilinear forms, which implies the monotonicity relationship

$$
\operatorname{Vol}_{R}\left(u_{1}, \ldots, u_{k}\right) \leq \operatorname{Vol}_{4 R}\left(u_{1}, \ldots, u_{k}\right) .
$$

When $k$ is large, and the $u_{1}, \ldots, u_{k}$ are approximately harmonic, we can improve this inequality by applying Proposition 7.3 on large balls and Proposition 7.2 on small balls. More precisely, we have the following inequality (cf. [Kl, Lem.3.16], [CM, Prop. 4.16]):

Proposition 7.4 (Volume decrease). Let $k \geq 1$ be an integer, $0<\varepsilon, \delta<1$ and $R>1 / \delta$. Let $u_{1}, \ldots, u_{k}: G \rightarrow \mathbf{R}$ be $\varepsilon$-harmonic Lipschitz functions. Suppose also that

$$
k \geq 2\left(\frac{\left|B_{S}(2 R)\right|}{\left|B_{S}(\delta R)\right|}+1\right)
$$

Then we have

$$
\begin{aligned}
& \operatorname{Vol}_{R}\left(u_{1}, \ldots, u_{k}\right) \\
& \leq O(|S|)^{O(k)}\left(\delta^{k / 2}\left(\frac{\left|B_{S}(7 \delta R)\right|}{\left|B_{S}(\delta R)\right|}\right)^{k / 2} \operatorname{Vol}_{4 R}\left(u_{1}, \ldots, u_{k}\right)+k \varepsilon^{2} R^{k+2}\left|B_{S}(4 R)\right|^{k / 2}\right) .
\end{aligned}
$$


Proof. Let us write

$$
v:=\operatorname{Vol}_{4 R}\left(u_{1}, \ldots, u_{k}\right) .
$$

We may of course assume that

$$
v>k \varepsilon^{2} R^{k+2}\left|B_{S}(4 R)\right|^{k / 2},
$$

otherwise we are done by (17). In particular, this implies that $u_{1}, \ldots, u_{k}$ are linearly independent. Let $V$ be the $k$-dimensional subspace of $\mathbf{R}^{G}$ spanned by the $u_{1}, \ldots, u_{k}$; the non-vanishing of $v$ implies that $V$ is a Hilbert space with respect to the bilinear form $Q_{4 R}$.

We will now study a subspace $V^{\prime}$ of $V$ consisting of "locally mean zero" functions. Let $x_{1}, \ldots, x_{m}$ be a maximal $2 \delta R$-separated subset of $B_{S}(R)$. Then the balls $B\left(x_{j}, \delta R\right)$ for $1 \leq j \leq m$ are disjoint and contained in $B(2 R)$, so we have the upper bound

$$
m \leq \frac{\left|B_{S}(2 R)\right|}{\left|B_{S}(\delta R)\right|} .
$$

We then introduce the subspace

$$
V^{\prime}:=\left\{u \in V: u_{B\left(x_{j}, 2 \delta R\right)}=0 \text { for all } 1 \leq j \leq m ; u(0)=0\right\},
$$

where we recall that $u_{B}:=\frac{1}{|B|} \sum_{y \in B} u(y)$ is the mean of $u$ on $B$. Clearly $V^{\prime}$ is a subspace of $V$ of codimension at most $m+1$; by (18), we conclude that

$$
\operatorname{dim}\left(V^{\prime}\right) \geq k / 2 .
$$

Using the Gram-Schmidt process, we may then find an orthonormal (with respect to $\left.Q_{R}\right)$ basis $\tilde{u}_{1}, \ldots, \tilde{u}_{k}$ of $V$ such that $\tilde{u}_{1}, \ldots, \tilde{u}_{\operatorname{dim}\left(V^{\prime}\right)}$ lies in $V^{\prime}$.

Let $1 \leq i \leq \operatorname{dim}\left(V^{\prime}\right)$. From Lemma 7.2 and the construction of $V^{\prime}$ we have

$$
\sum_{y \in B\left(x_{j}, 2 \delta R\right)}\left|\tilde{u}_{i}(y)\right|^{2} \ll \delta^{2} R^{2} \sum_{y \in B\left(x_{j}, 6 \delta R\right)}\left|\nabla \tilde{u}_{i}(y)\right|^{2} .
$$

Now suppose a point $x$ is contained in $J$ balls $B\left(x_{j}, 6 \delta R\right)$. Then the $J$ balls $B\left(x_{j}, \delta R\right)$ are contained in $B(x, 7 \delta R)$. Since these balls are disjoint, we conclude that $J \leq$ $\left|B_{S}(7 \delta R)\right| /\left|B_{S}(\delta R)\right|$. On the other hand, all the $B\left(x_{j}, 6 \delta R\right)$ are contained in $B_{S}(2 R)$. We can therefore sum $(22)$ in $j$ to conclude that

$$
\sum_{j=1}^{m} \sum_{y \in B\left(x_{j}, 2 \delta R\right)}\left|\tilde{u}_{i}(y)\right|^{2} \ll \delta^{2} R^{2} \frac{\left|B_{S}(7 \delta R)\right|}{\left|B_{S}(\delta R)\right|} \int_{y \in B_{S}(2 R)}\left|\nabla \tilde{u}_{i}(y)\right|^{2} .
$$

On the other hand, by construction of the $x_{j}$ we see that the balls $B\left(x_{j}, 2 \delta R\right)$ cover $B_{S}(R)$. Since $\tilde{u}_{i}(0)=0$, we thus see from (16) that

$$
Q_{R}\left(\tilde{u}_{i}\right) \ll \delta^{2} R^{2} \frac{\left|B_{S}(7 \delta R)\right|}{\left|B_{S}(\delta R)\right|} \sum_{y \in B_{S}(2 R)}\left|\nabla \tilde{u}_{i}(y)\right|^{2} .
$$

To estimate this, we wish to use Lemma 7.3 , but first we must express $\tilde{u}_{i}$ as an approximately harmonic function. As the $u_{j}$ have Lipschitz norm at most 1 , we see from (16) that

$$
Q_{R}\left(u_{j}, u_{j}\right) \leq R^{2}\left|B_{S}(R)\right|
$$


for all $1 \leq j \leq k$. Thus when represented in the orthonormal basis $\tilde{u}_{1}, \ldots, \tilde{u}_{k}$, the $u_{1}, \ldots, u_{k}$ are elements of $\mathbf{R}^{k}$ of norm at most $R\left|B_{S}(R)\right|^{1 / 2}$, whose coordinates form a $k \times k$ matrix whose determinant of magnitude $v$. Taking adjugates (i.e. using Cramer's rule), we conclude that the inverse of this matrix has coefficients of size $O\left(v^{-1}\left(R\left|B_{S}(R)\right|^{1 / 2}\right)^{k-1}\right)$. In other words, the $\tilde{u}_{1}, \ldots, \tilde{u}_{k}$ are linear combinations of the $u_{1}, \ldots, u_{k}$ whose coefficients have size $O\left(v^{-1}\left(R\left|B_{S}(R)\right|^{1 / 2}\right)^{k-1}\right)$. In particular, each $\tilde{u}_{i}$ is equal to $O\left(k v^{-1}\left(R\left|B_{S}(R)\right|^{1 / 2}\right)^{k-1}\right)$ times a $\varepsilon$-harmonic Lipschitz function (note that the property of being $\varepsilon$-harmonic Lipschitz is convex). We may then apply Lemma 7.3 to conclude that

$$
\sum_{y \in B_{S}(2 R)}\left|\nabla \tilde{u}_{i}(y)\right|^{2} \ll|S|^{O(1)}\left(\frac{1}{R^{2}} \sum_{y \in B_{S}(4 R)}\left|\tilde{u}_{i}(y)\right|^{2}+k \varepsilon^{2} v^{-1} R^{k}\left|B_{S}(4 R)\right|^{k / 2}\right) .
$$

Since $\tilde{u}_{i}(0)=0$, and $\tilde{u}_{i}$ is a unit vector with respect to $Q_{4 R}$, we see from (16) that

$$
\sum_{y \in B_{S}(4 R)}\left|\tilde{u}_{i}(y)\right|^{2}=1 .
$$

Inserting these estimates and (20) into (23) we obtain

$$
Q_{R}\left(\tilde{u}_{i}\right) \ll|S|^{O(1)} \delta^{2} \frac{\left|B_{S}(7 \delta R)\right|}{\left|B_{S}(\delta R)\right|}
$$

for $1 \leq i \leq \operatorname{dim}\left(V^{\prime}\right)$. Meanwhile, for $\operatorname{dim}\left(V^{\prime}\right)<i \leq m$ we have the crude bound

$$
Q_{R}\left(\tilde{u}_{i}\right) \leq Q_{4 R}\left(\tilde{u}_{i}\right)=1 \text {. }
$$

We conclude (using (21)) that

$$
\operatorname{Vol}_{R}\left(\tilde{u}_{1}, \ldots, \tilde{u}_{k}\right) \ll O(|S|)^{O(k)} \delta^{k / 2}\left(\frac{\left|B_{S}(7 \delta R)\right|}{\left|B_{S}(\delta R)\right|}\right)^{k / 2} .
$$

On the other hand, by orthonormality we have $\operatorname{Vol}_{4 R}\left(\tilde{u}_{1}, \ldots, \tilde{u}_{k}\right)$. Observe that the ratio $\operatorname{Vol}_{R}\left(u_{1}, \ldots, u_{k}\right) / \operatorname{Vol}_{4 R}\left(u_{1}, \ldots, u_{k}\right)$ is invariant under row operations, and therefore by (19) we have

$$
\operatorname{Vol}_{R}\left(u_{1}, \ldots, u_{k}\right) \ll O(|S|)^{O(k)} \delta^{k}\left(\frac{\left|B_{S}(7 \delta R)\right|}{\left|B_{S}(\delta R)\right|}\right)^{k} \operatorname{Vol}_{4 R}\left(u_{1}, \ldots, u_{k}\right),
$$

and the claim follows.

Now we iterate Proposition 7.4 to obtain

Proposition 7.5 (Volume bound). Let $k \geq 1$ be an integer, $0<\varepsilon<1$, $0<\kappa<0.1, d \geq 1$, and $R_{0}>1$. Assume that

$$
k \geq C^{d / \kappa}
$$

and

$$
R_{0} \geq C^{1 / \kappa} k^{C}
$$

for some sufficiently large constant $C$. Then for every $\left(R_{0}, d\right)$-growth group $(G, S)$, and every $\varepsilon$-harmonic Lipschitz functions $u_{1}, \ldots, u_{k}: G \rightarrow \mathbf{R}$, we have

$$
\operatorname{Vol}_{R_{0}^{1-\kappa}}\left(u_{1}, \ldots, u_{k}\right) \ll R_{0}^{O(k(d+\log |S|))-c \frac{\kappa^{2}}{d+1} k \log k}+\varepsilon^{2} O\left(R_{0}\right)^{O(k d)}
$$

for some absolute constant $c>0$. 
Proof. We choose $\delta$ by the solving the equation

$$
k=C^{\frac{d}{\kappa} \log \frac{1}{\delta}}
$$

for some large absolute constant $C$, thus

$$
\delta=k^{-\frac{\kappa}{d \log C}} .
$$

From our assumptions on $k, R_{0}$ we see (for sufficiently large choices of constants) that

$$
R_{0}^{-0.1}<\delta<0.1
$$

Let $n$ be the largest integer such that $4^{N} R_{0}^{1-\kappa} \leq R_{0}$, thus

$$
N \gg \kappa \log R_{0}
$$

(note from hypothesis that $\kappa \log R_{0}>1$ ). Set $R_{n}:=4^{n} R_{0}^{1-\kappa}$ for $1 \leq n \leq N$. By telescoping series and (1) we see that

$$
\prod_{n=1}^{N} \frac{\left|B_{S}\left(2 R_{n}\right)\right|}{\left|B_{S}\left(\delta R_{n}\right)\right|} \ll R_{0}^{O\left(d \log \frac{1}{\delta}\right)} \ll O(1)^{\frac{d N}{\kappa}} \log \frac{1}{\delta} .
$$

We thus conclude that for at least $N / 2$ values of $1 \leq n \leq N$, we have

$$
\frac{\left|B_{S}\left(2 R_{n}\right)\right|}{\left|B_{S}\left(\delta R_{n}\right)\right|} \leq O(1)^{\frac{d}{\kappa} \log \frac{1}{\delta}}
$$

By (25), we thus have (for $C$ large enough) that

$$
k \geq 2\left(\frac{\left|B_{S}\left(2 R_{n}\right)\right|}{\left|B_{S}\left(\delta R_{n}\right)\right|}+1\right)
$$

for at least $N / 2$ values of $n$. For each such $n$, we may apply Proposition 7.4 and conclude that

$$
\begin{aligned}
\operatorname{Vol}_{R_{n}}\left(u_{1}, \ldots, u_{k}\right) \leq O(|S|)^{O(k)} \delta^{k / 2} & \left(\frac{\left|B_{S}\left(7 \delta R_{n}\right)\right|}{\left|B_{S}\left(\delta R_{n}\right)\right|}\right)^{k / 2} \\
\cdot & \operatorname{Vol}_{R_{n+1}}\left(u_{1}, \ldots, u_{k}\right)+k \varepsilon^{2} R_{n}^{k+2}\left|B_{S}\left(4 R_{n}\right)\right|^{k / 2} .
\end{aligned}
$$

We may make the assumption

$$
\operatorname{Vol}_{R_{0}^{1-\kappa}}\left(u_{1}, \ldots, u_{k}\right)>2 k \varepsilon^{2} R_{0}^{k+2+d k / 2}
$$

since we are done otherwise. Then (by (1) and monotonicity of volume) we have

$$
\operatorname{Vol}_{R_{n}}\left(u_{1}, \ldots, u_{k}\right)>2 k \varepsilon^{2} R_{n}^{k+2}\left|B_{S}\left(2 R_{n}\right)\right|^{k / 2}
$$

and thus

$$
\operatorname{Vol}_{R_{n}}\left(u_{1}, \ldots, u_{k}\right) \leq O(|S|)^{O(k)} \delta^{k / 2}\left(\frac{\left|B_{S}\left(7 \delta R_{n}\right)\right|}{\left|B_{S}\left(\delta R_{n}\right)\right|}\right)^{k / 2} \operatorname{Vol}_{R_{n+1}}\left(u_{1}, \ldots, u_{k}\right)
$$

for at least $N / 2$ values of $n$. For the other values of $n$, we see from (17) that

$$
\operatorname{Vol}_{R_{n}}\left(u_{1}, \ldots, u_{k}\right) \leq \operatorname{Vol}_{R_{n+1}}\left(u_{1}, \ldots, u_{k}\right) \text {. }
$$


Putting this all together and using monotonicity of volume again, we conclude that

$$
\operatorname{Vol}_{R_{0}^{1-\kappa}}\left(u_{1}, \ldots, u_{k}\right) \leq O(1)^{k^{2} N}|S|^{k N} \delta^{k N / 4}\left(\prod_{n=0}^{N} \frac{\left|B_{S}\left(7 \delta R_{n}\right)\right|}{\left|B_{S}\left(\delta R_{n}\right)\right|}\right)^{k / 2} \operatorname{Vol}_{R_{0}}\left(u_{1}, \ldots, u_{k}\right) \text {. }
$$

On the other hand, by telescoping series and (1) we have

$$
\prod_{n=0}^{N} \frac{\left|B_{S}\left(7 \delta R_{n}\right)\right|}{\left|B_{S}\left(\delta R_{n}\right)\right|} \leq R_{0}^{O(d)}
$$

Also, arguing as in the proof (24) we have

$$
Q_{R_{0}}\left(u_{i}, u_{i}\right) \leq R_{0}^{2}\left|B_{S}(R)\right| \leq R_{0}^{d+2}
$$

and thus

$$
\operatorname{Vol}_{R_{0}}\left(u_{1}, \ldots, u_{k}\right) \leq R_{0}^{O(k d)}
$$

We conclude that

$$
\operatorname{Vol}_{R_{0}^{1-\kappa}}\left(u_{1}, \ldots, u_{k}\right) \leq O(|S|)^{k N} \delta^{k N / 4} R_{0}^{O(k d)} .
$$

Substituting in (26), (28) we obtain the claim.

In practice, we will only use this proposition in the regime where $d, 1 / \kappa,|S|$ are bounded, $k$ is sufficiently large depending on these parameters (but independent of $R_{0}$ ), and $\varepsilon$ is less than extremely large negative power of $R_{0}$. More precisely, we will use the following corollary of Proposition 7.5:

Corollary 7.6 (Volume bound). Let $0<\kappa<0.1, d>0, R_{0}>1$, and let $(G, S)$ be a $\left(R_{0}, d\right)$-growth group. Suppose that

$$
k \geq(C|S|)^{C d^{3} / \kappa^{2}}
$$

and

$$
R_{0} \geq k^{C}
$$

for some sufficiently large absolute constant $C$. Then for any $R_{0}^{-C k d}$-harmonic Lipschitz functions $u_{1}, \ldots, u_{k}: G \rightarrow \mathbf{R}$, we have

$$
\operatorname{Vol}_{R_{0}^{1-\kappa}}\left(u_{1}, \ldots, u_{k}\right) \leq R_{0}^{-100 k d} .
$$

Now we can prove Theorem 7.1. We first observe that it suffices to prove Theorem 7.1 for $R_{0}^{-K}$-harmonic Lipschitz functions which vanish at the identity, since the general case can then be handled by adding the constant functions to $V$ (increasing the dimension by one). By Corollary 7.6 and the hypothesis on $R_{0}$, we can find (if $C$ is large enough) a threshold $k_{0}=O(|S|)^{O\left((1+d)^{3} / \kappa^{2}\right)}$ such that $\operatorname{Vol}_{R_{0}^{1-\kappa}}\left(u_{1}, \ldots, u_{k_{0}}\right) \leq R_{0}^{-100 k_{0} d}$ for all $R_{0}^{-K}$-harmonic Lipschitz functions $u_{1}, \ldots, u_{k_{0}}: G \rightarrow \mathbf{R}$. Using the greedy algorithm, one may then find $R_{0}^{-K}$-harmonic Lipschitz functions $u_{1}, \ldots, u_{k}: G \rightarrow \mathbf{R}$ for some $0 \leq k<k_{0}$ such that

$$
\operatorname{Vol}_{R_{0}^{1-\kappa}}\left(u_{1}, \ldots, u_{k}, u\right) \leq R_{0}^{-100 d} \operatorname{Vol}_{R_{0}^{1-\kappa}}\left(u_{1}, \ldots, u_{k}\right)
$$

for all $R_{0}^{-K}$-harmonic Lipschitz functions $u: G \rightarrow \mathbf{R}$. But if we let $V$ be the space spanned by $u_{1}, \ldots, u_{k}$, then from the base times height formula we have

$$
\operatorname{Vol}_{R_{0}^{1-\kappa}}\left(u_{1}, \ldots, u_{k}, u\right)=\operatorname{dist}_{Q_{R_{0}^{1-\kappa}}}(u, V) \operatorname{Vol}_{R_{0}^{1-\kappa}}\left(u_{1}, \ldots, u_{k}\right)
$$

and the claim follows. 


\section{Third Step for Proposition 5.2: Establishing an Approximate Isometric Representation}

The group $G$ acts on the space $\mathbf{R}^{G}$ of functions $u: G \rightarrow \mathbf{R}$ by left translation, thus

$$
\rho(g)(u)(x):=u\left(g^{-1} x\right)
$$

for all $u \in \mathbf{R}^{G}, g \in G, x \in G$. Observe that this action preserves the vector space $V$ of Lipschitz harmonic functions. In view of Kleiner's theorem, and assuming $G$ has polynomial growth, this gives a finite-dimensional linear representation of $G$; on the quotient space $V / \mathbf{R}$ of $V$ modulo the constant functions, the Lipschitz semi-norm becomes a norm, which is preserved by the group action.

The purpose of this section is to establish an analogous claim for $\left(R_{0}, d\right)$ groups rather than groups of polynomial growth. It is convenient to work modulo the constants. Let $\mathbf{R}^{G} / \mathbf{R}$ be the space of functions from $G$ to $\mathbf{R}$ modulo addition by constants, and let $\pi: \mathbf{R}^{G} \rightarrow \mathbf{R}^{G} / \mathbf{R}$ be the quotient map. Observe that the action $\rho$ of $G$ on $\mathbf{R}^{G}$ descends to an action $\bar{\rho}$ on $\mathbf{R}^{G} / \mathbf{R}$. One can also meaningfully define the concept of a $\varepsilon$-harmonic Lipschitz function $\bar{u}$ in $\mathbf{R}^{G} / \mathbf{R}$, since this concept is invariant under addition by constants. We can also define induced $\overline{\ell^{p}(B)}$ norms for every finite $B \subset G$ by

$$
\|\bar{u}\|_{\ell_{\ell^{p}(B)}}:=\inf \left\{\|u\|_{\ell^{p}(B)}: \pi(u)=\bar{u}\right\}
$$

for any $\bar{u} \in \mathbf{R}^{G} / \mathbf{R}$. For $p=2$, this norm is associated with an inner product

$$
\langle\bar{u}, \bar{v}\rangle_{\overline{\ell^{2}(B)}}=\sum_{y \in B} u(y) v(y)
$$

where $u, v$ are the unique lifts of $\bar{u}, \bar{v}$ by $\pi$ that have mean zero on $B$.

We will need a quotiented variant of Theorem 7.1 with an additional "good scale" $R_{1}$ which is stable under translations.

Proposition 8.1 (Quantitative Kleiner theorem with good scale). Let $0<\kappa<0.1$, and let $(G, S)$ be a $\left(R_{0}, d\right)$-growth group with $d \geq 1$ obeying

$$
R_{0} \geq K:=(C|S|)^{C d^{3} / \kappa^{3}}
$$

for some sufficiently large $C$. Then there exists a finite-dimensional subspace $\bar{V}$ of $\mathbf{R}^{G} / \mathbf{R}$ of dimension

$$
\operatorname{dim}(\bar{V}) \leq O(|S|)^{O\left(d^{3} / \kappa^{2}\right)}
$$

and a scale $R_{0}^{1-2 \kappa} \leq R_{1} \leq R_{0}^{1-\kappa}$ with two properties:

- Every $R_{0}^{-K}$-harmonic Lipschitz function $\bar{u} \in \mathbf{R}^{G} / \mathbf{R}$ lies at a distance at most $R_{0}^{-99 d}$ from $\bar{V}$ in the $\overline{\ell^{2}\left(B_{S}\left(R_{1}\right)\right)}$ norm.

- For every $\bar{u} \in \bar{V}$, we have

$$
\|\bar{u}\|_{\ell^{2}\left(B_{S}\left(R_{1}\right)\right)} \leq\left(1+R_{0}^{-\kappa}\right)\|\bar{u}\|_{\ell^{2}\left(B_{S}\left(R_{1}-R_{0}^{1-4 \kappa}\right)\right)} .
$$

Proof. We first make the observation that we may replace (31) by the variant condition

$$
\|\bar{u}\|_{\ell^{2}\left(B_{S}\left(R_{1} / 2\right)\right)} \geq R_{0}^{-200 d}\|\bar{u}\|_{\ell^{2}\left(B_{S}\left(R_{1}\right)\right)}
$$


at the slight cost of tightening the lower bound $R_{1} \geq R_{0}^{1-2 \kappa}$ to $R_{1} \geq 2 R_{0}^{1-2 \kappa}$. Indeed, suppose that (32) held for all $\bar{u} \in \bar{V}$. Then, if we let $Q_{r}: V \rightarrow \mathbf{R}$ denote the positive definite quadratic form $Q_{r}(\bar{u}):=\|\bar{u}\|_{\ell^{2}\left(B_{S}(r)\right)}^{2}$ for each $r>0$, we see that $\operatorname{det} Q_{R_{1}} / \operatorname{det} Q_{R_{1} / 2} \leq\left(R_{0}^{200 d}\right)^{2 \operatorname{dim}(\bar{V})}$ (where we pick some arbitrary fixed basis of $V$ with which to compute determinants). As the $Q_{r}$ are increasing in $r$, we may then use the pigeonhole principle (and the bounds on $R_{0}$ and $\operatorname{dim}(\bar{V})$ ) and find $R_{1} / 2 \leq R_{1}^{\prime} \leq R_{1}$ such that

$$
\operatorname{det} Q_{R_{1}^{\prime}} \leq\left(1+R_{0}^{-\kappa}\right)^{2} \operatorname{det} Q_{R_{1}^{\prime}-R_{0}^{1-4 \kappa}}
$$

diagonalizing the quadratic form $Q_{R_{1}^{\prime}}$ with respect to $Q_{R_{1}^{\prime}-R_{0}^{1-4 \kappa}}$ we obtain (31) with $R_{1}$ replaced by the slightly smaller $R_{1}^{\prime}$.

It remains to find a $\bar{V}$ and $R_{1}$ obeying (32), as well as the property about $\bar{V}$ approximating $R_{0}^{-K}$-harmonic Lipschitz functions. From Theorem 7.1 and a quotienting by $\pi$, we see that if we (temporarily) set $R_{1}:=R_{0}^{1-\kappa}$, we may find a subspace $\bar{V}_{0}$ of $\mathbf{R}^{G} / \mathbf{R}$ of dimension $O(|S|)^{O\left(d^{3} / \kappa^{2}\right)}$ such that every $R_{0}^{-K}$-harmonic Lipschitz function $\bar{u} \in \mathbf{R}^{G} / \mathbf{R}$ lies at a distance at most $R_{0}^{-100 d}$ from $\bar{V}_{0}$ in the $\overline{\ell^{2}\left(B_{S}\left(R_{1}\right)\right)}$ norm.

If the property (32) holds for all $\bar{u} \in \bar{V}_{0}$ with this choice of $R_{1}$, then we are done. Otherwise, suppose there exists $\bar{u}_{0} \in \bar{V}_{0}$ for which

$$
\left\|\bar{u}_{0}\right\|_{\ell^{2}\left(B_{S}\left(R_{1} / 2\right)\right)}<R_{0}^{-200 d}\left\|\bar{u}_{0}\right\|_{\ell^{2}\left(B_{S}\left(R_{1}\right)\right)}
$$

Clearly, $\bar{u}_{0}$ is non-zero; we may normalize

$$
\left\|\bar{u}_{0}\right\|_{\ell^{2}\left(B_{S}\left(R_{1}\right)\right)}=1 .
$$

Let $\bar{V}_{1}$ be the orthogonal complement of $\bar{u}_{0}$ in $\bar{V}_{0}$, thus $\bar{V}_{1}$ has dimension one less than $\bar{V}_{0}$.

Now let $\bar{u} \in \mathbf{R}^{G} / \mathbf{R}$ be a $R_{0}^{-K}$-harmonic Lipschitz function. By construction, we can find $\bar{v} \in \bar{V}_{0}$ such that

$$
\|\bar{u}-\bar{v}\|_{\ell^{2}\left(B_{S}\left(R_{1}\right)\right)} \leq R_{0}^{-100 d} .
$$

On the other hand, $\bar{u}$ has Lipschitz constant at most 1 and thus (since constants have been quotiented out)

In particular

$$
\|\bar{u}\|_{\ell^{2}\left(B_{S}\left(R_{1}\right)\right)} \leq R_{1}\left|B_{S}\left(R_{1}\right)\right|^{1 / 2} \leq R_{0}^{1+d / 2}
$$

$$
\|\bar{v}\|_{\ell^{2}\left(B_{S}\left(R_{1}\right)\right)} \ll R_{1}^{1+d / 2}
$$

and hence by Cauchy-Schwarz and (34)

$$
\left\langle\bar{v}, \bar{u}_{0}\right\rangle \frac{}{\ell^{2}\left(B_{S}\left(R_{1}\right)\right)}=O\left(R_{0}^{1+d / 2}\right) .
$$

We split $\bar{v}=\bar{v}_{1}+\left\langle\bar{v}, \bar{u}_{0}\right\rangle_{\ell^{2}\left(B_{S}\left(R_{1}\right)\right)} \overline{u_{0}}$, where $\bar{v}_{1} \in \bar{V}_{1}$ is the orthogonal projection of $\bar{v}$ to $\bar{V}_{1}$. From (33), (34), (36) the latter term is small on $B_{S}\left(R_{1} / 2\right)$ :

$$
\left\|\left\langle\bar{v}, \bar{u}_{0}\right\rangle_{\ell^{2}\left(B_{S}\left(R_{1}\right)\right)} \overline{u_{0}}\right\|_{\ell^{2}\left(B_{S}\left(R_{1} / 2\right)\right)} \leq R_{0}^{-150 d}
$$


(say). From this, (35), and the triangle inequality we conclude that

$$
\left\|\bar{u}-\bar{v}_{1}\right\|_{\ell^{2}\left(B_{S}\left(R_{1} / 2\right)\right)} \leq R_{0}^{-100 d}+R_{0}^{-150 d} .
$$

Thus, the property that $\bar{V}_{0}$ approximates $R_{0}^{-K}$-harmonic Lipschitz functions has been inherited by $\bar{V}_{1}$, at the slight cost of reducing the scale $R_{1}$ to $R_{1} / 2$ and increasing the error of approximation slightly from $R_{0}^{-100 d}$ to $R_{0}^{-100 d}+R_{0}^{-150 d}$. One can then iterate this process at most $\operatorname{dim}\left(\bar{V}_{0}\right)=O(|S|)^{O\left(d^{3} / \kappa^{2}\right)}$ times until we find a space $\bar{V}$ and a scale $R_{1}$ for which (32) is satisfied; the dimension bound and the largeness hypothesis on $R_{0}$ ensures that $R_{1} \geq R_{0}^{1-2 \kappa}$, and that the total error of approximation never exceeds $R_{0}^{-99 d}$. The claim follows.

Let $\kappa,(G, S), R_{0}, d, K, \bar{V}, R_{1}$ be as in Proposition 8.1, and set $D:=\operatorname{dim}(\bar{V})$, thus

$$
D=O(|S|)^{O\left(d^{3} / \kappa^{2}\right)} \text {. }
$$

In practice, $D$ should be viewed as bounded (especially when compared with the large parameter $R_{0}$ ), and so the factors of $D^{O(1)}$ that appear below should be ignored at a first reading.

Let $\Omega \subset \bar{V}$ be the set of all elements of $\bar{V}$ which lie within a distance $R_{0}^{-99 d}$ in $\overline{\ell^{2}\left(B_{S}\left(R_{1}\right)\right)}$ norm from a $R_{0}^{-K}$-harmonic Lipschitz function. This is a symmetric convex subset of $\bar{V}$ with non-empty interior. Applying John's theorem $[\mathrm{J}]$, we may then find an ellipsoid $E \subset \bar{V}$ such that $E \subset \Omega \subset \sqrt{D} \cdot E$. If we let $e_{1}, \ldots, e_{D} \in \bar{V}$ and $\lambda_{1}, \ldots, \lambda_{D}>0$ be the principal orthonormal directions and radii of this ellipsoid with respect to the Hilbert space structure $\overline{\ell^{2}\left(B_{S}\left(R_{1}\right)\right)}$ on $\bar{V}$, we thus see that

$$
\lambda_{i} e_{i} \in \Omega
$$

for $i=1, \ldots, D$, and conversely every element of $\Omega$ can be represented in the form

$$
\sum_{i=1}^{D} t_{i} \lambda_{i} e_{i}
$$

for some $t_{1}, \ldots, t_{D}=O\left(D^{O(1)}\right)$.

Since $\Omega$ (and hence $\sqrt{D} \cdot E$ ) contains the ball of radius $R_{0}^{-99 d}$, we have the lower bound

$$
\lambda_{i} \geq R_{0}^{-99 d} / \sqrt{D}
$$

for all $1 \leq i \leq D$. Also since $R_{0}^{-K}$-harmonic Lipschitz functions have an $\overline{\ell^{2}\left(B_{S}\left(R_{1}\right)\right)}$ norm of at most $R_{1}\left|B_{S}\left(R_{1}\right)\right|^{1 / 2} \leq R_{0}^{(d+1) / 2}$, we have the upper bound

$$
\lambda_{i} \ll R_{0}^{(d+1) / 2}
$$

for $1 \leq i \leq D$.

Let $1 \leq i \leq D$ and $g \in G$. By construction, there exists a $R_{0}^{-K}$-harmonic Lipschitz function $\bar{u}_{i} \in \mathbf{R}^{G} / \mathbf{R}$ with $\left\|\overline{u_{i}}-\lambda_{i} e_{i}\right\|_{\ell^{2}\left(B_{S}\left(R_{1}\right)\right)} \leq R_{0}^{-99 d}$. Translating this, we obtain

$$
\left\|\bar{\rho}(g) \overline{u_{i}}-\lambda_{i} \bar{\rho}(g) e_{i}\right\|_{\ell^{2}\left(g \cdot B_{S}\left(R_{1}\right)\right)} \leq R_{0}^{-99 d} .
$$

In particular, if $g \in B_{S}\left(R_{0}^{1-5 \kappa}\right)$, then

$$
\left\|\bar{\rho}(g) \overline{u_{i}}-\lambda_{i} \bar{\rho}(g) e_{i}\right\|_{\ell^{2}\left(B_{S}\left(R_{1}-R_{0}^{1-5 \kappa}\right)\right)} \leq R_{0}^{-99 d} .
$$


On the other hand, $\bar{\rho}(g) \overline{u_{i}}$ is also a $R_{0}^{-K}$-harmonic Lipschitz function, and thus must lie within $R_{0}^{-99 d}$ in $\ell^{2}\left(B_{S}\left(R_{1}\right)\right)$ norm of some function $f_{g, i} \in \Omega$. By the triangle inequality we thus have

$$
\left\|f_{g, i}-\lambda_{i} \bar{\rho}(g) e_{i}\right\|_{\ell^{2}\left(B_{S}\left(R_{1}-R_{0}^{1-5 \kappa}\right)\right)} \leq 2 R_{0}^{-99 d} .
$$

Using (38), we can write

$$
f_{g, i}=\sum_{j=1}^{D} t_{g, j, i} \lambda_{j} e_{j}
$$

for some $t_{g, i, j}$ obeying the bounds

$$
\left|t_{g, j, i}\right| \ll D^{O(1)} .
$$

Another bound on these coefficients is obtained by observing that

$$
\left\|\lambda_{i} \bar{\rho}(g) e_{i}\right\|_{\ell^{2}\left(B_{S}\left(R_{1}-R_{0}^{1-5 \kappa}\right)\right)} \leq \lambda_{i}\left\|e_{i}\right\|_{\ell^{2}\left(B_{S}\left(R_{1}\right)\right)}=\lambda_{i}
$$

and thus (by (41), (39))

$$
\left\|f_{g, i}\right\|_{\ell^{2}\left(B_{S}\left(R_{1}-R_{0}^{1-5 \kappa}\right)\right)} \ll D^{O(1)} \lambda_{i}
$$

and thus (by (31))

$$
\left\|f_{g, i}\right\|_{\ell^{2}\left(B_{S}\left(R_{1}\right)\right)} \ll D^{O(1)} \lambda_{i} .
$$

Using (42) and the orthonormal properties of $e_{j}$ we conclude that

$$
\left|t_{g, j, i}\right| \ll D^{O(1)} \lambda_{i} / \lambda_{j}
$$

We now investigate the extent to which the $D \times D$ matrices $U_{g}:=\left(t_{g, j, i}\right)_{1 \leq j, i \leq D}$ behave like a representation. From construction we see that we may take $U_{\text {id }}=I$, where $I$ is the $D \times D$ matrix. Now we look at the multiplicativity.

Proposition 8.2 ( $U_{g}$ approximately multiplicative). If $g, h \in B_{S}\left(R_{0}^{1-5 \kappa} / 2\right)$ and $1 \leq i, k \leq D$, then the $(k, i)$ entry of the matrix $U_{g h}-U_{g} U_{h}$, i.e.

$$
t_{g h, k, i}-\sum_{j=1}^{d} t_{g, k, j} t_{h, j, i}
$$

has magnitude $O\left(D^{O(1)} R_{0}^{-99 d} / \lambda_{k}\right)$.

Proof. From (41), (42) we have

$$
\left\|\sum_{j=1}^{D} t_{h, j, i} \lambda_{j} e_{j}-\lambda_{i} \bar{\rho}(h) e_{i}\right\|_{\ell^{2}\left(B_{S}\left(R_{1}-R_{0}^{1-5 \kappa}\right)\right)} \leq 2 R_{0}^{-99 d}
$$

for all $1 \leq i \leq D$; applying $\bar{\rho}(g)$ to this, we conclude

$$
\left\|\sum_{j=1}^{D} t_{h, j, i} \lambda_{j} \bar{\rho}(g) e_{j}-\lambda_{i} \bar{\rho}(g h) e_{i}\right\|_{\ell^{2}\left(B_{S}\left(R_{1}-2 R_{0}^{1-5 \kappa}\right)\right)} \leq 2 R_{0}^{-99 d} .
$$

Meanwhile, from (41), (42) we have

$$
\left\|\lambda_{j} \bar{\rho}(g) e_{j}-\sum_{k=1}^{D} t_{g, k, j} \lambda_{k} e_{k}\right\|_{\ell^{2}\left(B_{S}\left(R_{1}-2 R_{0}^{1-5 \kappa}\right)\right)} \leq 2 R_{0}^{-99 d},
$$


and thus by the triangle inequality we have

$$
\left\|\lambda_{i} \bar{\rho}(g h) e_{i}-\sum_{k=1}^{D} \sum_{j=1}^{D} t_{h, j, i} t_{g, k, j} \lambda_{k} e_{k}\right\|_{\ell^{2}\left(B_{S}\left(R_{1}-2 R_{0}^{1-5 \kappa}\right)\right)} \ll D^{O(1)} R_{0}^{-99 d} .
$$

Meanwhile, from one final application of (41), (42) we have

$$
\left\|\lambda_{i} \bar{\rho}(g h) e_{i}-\sum_{k=1}^{D} t_{g h, k, i} \lambda_{k} e_{k}\right\|_{\ell^{2}\left(B_{S}\left(R_{1}-2 R_{0}^{1-5 \kappa}\right)\right)} \ll D^{O(1)} R_{0}^{-99 d}
$$

and so by the triangle inequality

$$
\left\|\sum_{k=1}^{D}\left[t_{g h, k, i}-\sum_{j=1}^{d} t_{h, j, i} t_{g, k, j}\right] \lambda_{k} e_{k}\right\|_{\ell^{2}\left(B_{S}\left(R_{1}-2 R_{0}^{1-5 \kappa}\right)\right)} \ll D^{O(1)} R_{0}^{-99 d},
$$

and thus by (31)

$$
\left\|\sum_{k=1}^{D}\left[t_{g h, i, k}-\sum_{j=1}^{d} t_{h, i, j} t_{g, j, k}\right] \lambda_{k} e_{k}\right\|_{\ell^{2}\left(B_{S}\left(R_{1}\right)\right)} \ll D^{O(1)} R_{0}^{-99 d} .
$$

As the $e_{k}$ are orthonormal, the claim follows.

\section{Fourth Step for Proposition 5.2: Taking Commutators}

We continue the discussion in the previous section. To simplify the expressions slightly we will make the smallness assumption

$$
\kappa \leq \frac{1}{d}, \frac{1}{\log |S|}
$$

on $\kappa$, and in particular from $(37)$

$$
D \leq 2^{O\left(\kappa^{-O(1)}\right)}
$$

For reasons that will be clearer later, we will also need to make $R_{0}$ larger than previously assumed, in particular we assume that

$$
R_{0} \geq 2^{2^{C / \kappa^{C}}}
$$

for some sufficiently large absolute constant $C$. (In particular, quantities such as $R_{0}^{\kappa^{10} / 2 D^{2}}$ are still quite large.)

The next step is to locate a large set of group elements $g \in G$ (which will be commutators of other group elements) for which $U_{g}$ are very close to the identity matrix $I$. The key point here is that if $U_{g}, U_{h}$ are within $\varepsilon$ of $I$ for some small $\varepsilon>0$ (in some suitable matrix norm), then the commutator $\left[U_{g}, U_{h}\right]$ is within $O\left(|D|^{O(1)} \varepsilon^{2}\right)$ of $I$. Meanwhile, from $(8.2)$, we expect $\left[U_{g}, U_{h}\right] \approx U_{[g, h]}$. The strategy here can be viewed as a simplified variant of the argument used to prove the Solovay-Kitaev theorem [KiSV].

We turn to the details. Let us write $\mathcal{O}_{\lambda}(X)$ to denote any matrix whose $(k, i)$ entry is $O\left(X / \lambda_{k}\right)$, and $\mathcal{O}_{\lambda}^{\lambda}(X)$ to denote any matrix whose $(k, i)$ entry is 
$O\left(X \min \left(1, \lambda_{i} / \lambda_{k}\right)\right)$. Then we can rewrite the conclusion of Proposition 8.2 as

$$
U_{g h}=U_{g} U_{h}+\mathcal{O}_{\lambda}\left(D^{O(1)} R_{0}^{-99 d}\right),
$$

and rewrite (44), (43) as

$$
U_{g}=\mathcal{O}_{\lambda}^{\lambda}\left(D^{O(1)}\right)
$$

We also observe the multiplication laws

$$
\mathcal{O}_{\lambda}^{\lambda}(X) \mathcal{O}_{\lambda}^{\lambda}(Y)=\mathcal{O}_{\lambda}^{\lambda}\left(D^{O(1)} X Y\right) ; \quad \mathcal{O}_{\lambda}^{\lambda}(X) \mathcal{O}_{\lambda}(Y), \mathcal{O}_{\lambda}(X) \mathcal{O}_{\lambda}^{\lambda}(Y)=\mathcal{O}_{\lambda}\left(D^{O(1)} X Y\right)
$$

In particular we have

$$
U_{g} U_{g^{-1}}, U_{g^{-1}} U_{g}=I+\mathcal{O}_{\lambda}\left(D^{O(1)} R_{0}^{-99 d}\right)
$$

for all $g \in B_{S}\left(R_{0}^{1-5 \kappa} / 2\right)$.

We have the following fundamental fact:

LEMmA 9.1 (Commutator bound). If $g, e, e^{\prime} \in B_{S}\left(R_{0}^{1-5 \kappa} / 100\right)$ and $0<\varepsilon \leq 1$ are such that

$$
U_{e}, U_{e^{\prime}}=I+\mathcal{O}_{\lambda}^{\lambda}(\varepsilon)+\mathcal{O}_{\lambda}(X),
$$

for some $R_{0}^{-99 d} \leq X \leq R_{0}^{-98 d}$, then

$$
U_{g\left[e, e^{\prime}\right] g^{-1}}=I+\mathcal{O}_{\lambda}^{\lambda}\left(D^{O(1)} \varepsilon^{2}\right)+\mathcal{O}_{\lambda}\left(D^{O(1)} X\right) .
$$

Proof. From (48), (49), (50) we have

$$
U_{\left[e, e^{\prime}\right]}=U_{e} U_{e^{\prime}} U_{e^{-1}} U_{\left(e^{\prime}\right)^{-1}}+\mathcal{O}_{\lambda}\left(D^{O(1)} X\right) .
$$

Splitting $U_{e^{\prime}}=\left(U_{e^{\prime}}-I\right)+I$ and using (51), (49), (50) we conclude that

$$
U_{\left[e, e^{\prime}\right]}=U_{e}\left(U_{e^{\prime}}-I\right) U_{e^{-1}} U_{\left(e^{\prime}\right)^{-1}}+U_{\left(e^{\prime}\right)^{-1}}+\mathcal{O}_{\lambda}\left(D^{O(1)} X\right) .
$$

From (52), (50) we have

$$
\left(U_{e}-I\right)\left(U_{e^{\prime}}-I\right),\left(U_{e^{\prime}}-I\right)\left(U_{e}-I\right)=\mathcal{O}_{\lambda}^{\lambda}\left(D^{O(1)} \varepsilon^{2}\right)+\mathcal{O}_{\lambda}\left(D^{O(1)} X\right),
$$

and thus

$$
U_{e}\left(U_{e^{\prime}}-I\right)=\left(U_{e^{\prime}}-I\right) U_{e}+\mathcal{O}_{\lambda}^{\lambda}\left(D^{O(1)} \varepsilon^{2}\right)+\mathcal{O}_{\lambda}\left(D^{O(1)} X\right) .
$$

Inserting this into (53) and using (48), (49), (50) we conclude that

$$
U_{\left[e, e^{\prime}\right]}=\left(U_{e^{\prime}}-I\right) U_{\left(e^{\prime}\right)^{-1}}+U_{\left(e^{\prime}\right)^{-1}}+\mathcal{O}_{\lambda}^{\lambda}\left(D^{O(1)} \varepsilon^{2}\right)+\mathcal{O}_{\lambda}\left(D^{O(1)} X\right) .
$$

Applying (53) again we obtain

$$
U_{\left[e, e^{\prime}\right]}=I+\mathcal{O}_{\lambda}^{\lambda}\left(D^{O(1)} \varepsilon^{2}\right)+\mathcal{O}_{\lambda}\left(D^{O(1)} X\right) .
$$

If we multiply this on the left by $U_{g}$ and on the right by $U_{g^{-1}}$ and use (51), (49), (50) we obtain the claim.

We would like to iterate this bound to find many $g$ with $U_{g}$ very close (e.g. $\left.O\left(R_{0}^{-50 d}\right)\right)$ to $I$, but to get started we will need to locate a preliminary supply of $g$ for which $U_{g}$ is somewhat close (e.g. $O\left(R_{0}^{-\kappa / 2 D^{2}}\right)$ ) to $I$. Morally, this should follow from the Dirichlet box principle (i.e. the pigeonhole principle) since the $U_{g}$ (and $U_{g^{-1}}$ ) are morally localized to a compact set of matrices (thanks to (49)) and are approximately multiplicative. We now make this intuition precise. 
Lemma 9.2 (Box principle). There exists a subgroup $\left(G^{\prime}, S^{\prime}\right)$ of $G$ with $S^{\prime} \subset B_{S}\left(R_{0}^{\kappa^{10}}\right)$ and finite index $\left|G: G^{\prime}\right| \leq R_{0}^{\kappa^{10}}$ such that

$$
U_{e}=I+\mathcal{O}_{\lambda}^{\lambda}\left(D^{O(1)} R_{0}^{-\kappa^{10} / 2 D^{2}}\right)+\mathcal{O}_{\lambda}\left(D^{O(1)} R_{0}^{-99 d}\right)
$$

for all $e \in S^{\prime}$.

Proof. From (49), the matrices $U_{g}$ for $g \in B_{S}\left(R_{0}^{1-5 \kappa} / 2\right)$ are contained in a set of matrices the form $\left\{U: U=\mathcal{O}_{\lambda}^{\lambda}\left(D^{O(1)}\right)\right\}$. From the convexity of the conditions used in the $\mathcal{O}_{\lambda}^{\lambda}\left(\right.$ ) notation (and the largeness hypothesis on $R_{0}$ ), we may cover this $D^{2}$-dimensional set by $M \leq R_{0}^{\kappa^{10}}$ balls $B_{1}, \ldots, B_{M}$ of the form $B_{m}=$ $\left\{U: U=U_{m}+\mathcal{O}_{\lambda}^{\lambda}\left(R^{-\kappa^{10} / 2 D^{2}}\right)\right\}$ for some $U_{m}=\mathcal{O}_{\lambda}^{\lambda}\left(D^{O(1)}\right)$. (Here we are using (47) to clean up the bounds somewhat.)

For each $r>0$, let $A_{r} \subset\{1, \ldots, M\}$ be the set of those $1 \leq m \leq M$ such that $U_{g} \in B_{m}$ for some $g \in B_{S}(r)$. Clearly the $A_{r}$ are increasing in $r$, so by the pigeonhole principle there exists $1 \leq r \leq M$ such that $A_{r+1}=A_{r}$.

Fix this $r$. For each $m \in A_{r}$, let $g_{m} \in B_{S}(r)$ be a representative such that $U_{g_{m}} \in B_{m}$. Since $A_{r+1}=A_{r}$, we see that for each $g \in B_{S}(r+1)$ there exists $m \in A_{r}$ such that $U_{g} \in B_{m}$, and in particular

$$
U_{g}=U_{g_{m}}+\mathcal{O}_{\lambda}^{\lambda}\left(R_{0}^{-\kappa^{10} / 2 D^{2}}\right) .
$$

Multiplying by $U_{g_{m}^{-1}}$ on the right and using (51), (49), (50), we see that

$$
U_{g g_{m}^{-1}}=I+\mathcal{O}_{\lambda}^{\lambda}\left(D^{O(1)} R_{0}^{-\kappa^{10} / 2 D^{2}}\right)+\mathcal{O}_{\lambda}\left(D^{O(1)} R_{0}^{-99 d}\right),
$$

and similarly

$$
U_{g_{m} g^{-1}}=I+\mathcal{O}_{\lambda}^{\lambda}\left(D^{O(1)} R_{0}^{-\kappa^{10} / 2 D^{2}}\right)+\mathcal{O}_{\lambda}\left(D^{O(1)} R_{0}^{-99 d}\right)
$$

Let $S^{\prime}$ denote the set of all $g g_{m}^{-1}, g_{m} g^{-1}$ that arise in this manner, then $S^{\prime} \subset B_{S}(2 r+1)$ is symmetric and

$$
B_{S}(r+1) \subset S^{\prime} \cdot\left\{g_{1}, \ldots, g_{M}\right\} \subset S^{\prime} \cdot B_{S}(r) .
$$

Iterating this we see that

$$
B_{S}(r+n) \subset B_{S^{\prime}}(n) \cdot B_{S}(r) \subset B_{S^{\prime}}(n+1) \cdot\left\{g_{1}, \ldots, g_{M}\right\}
$$

for all $n$; thus if $G^{\prime}$ denotes the group generated by $S^{\prime}$, then on taking unions as $n \rightarrow \infty$ we conclude that

$$
G \subset G^{\prime} \cdot\left\{g_{1}, \ldots, g_{m}\right\} .
$$

Thus $G^{\prime}$ has index at most $M$, and the claim follows.

Write $\varepsilon:=R_{0}^{-\kappa^{10} / 2 D^{2}}$, thus

$$
U_{e}=I+\mathcal{O}_{\lambda}^{\lambda}\left(D^{O(1)} \varepsilon\right)+\mathcal{O}_{\lambda}\left(D^{O(1)} R_{0}^{-99 d}\right)
$$

for all $e \in S^{\prime}$.

Since $(G, S)$ is a $\left(R_{0}, d\right)$-growth group and $S^{\prime} \subset B_{S}\left(R_{0}^{\kappa^{10}}\right)$, we see that $\left(G^{\prime}, S^{\prime}\right)$ is a $\left(R_{0}^{1-\kappa^{10}}, d /\left(1-\kappa^{10}\right)\right)$-growth group. Applying Lemma 4.7, we see that the commutator group $\left(G^{\prime}\right)^{(2)}:=\left[G^{\prime}, G^{\prime}\right]$ can be generated by a set $\left(S^{\prime}\right)^{(2)}$ of generators in 
$B_{S^{\prime}}\left(R_{0}^{\kappa^{10}}\right)$ of the form $g\left[e, e^{\prime}\right] g^{-1}$ for some $e, e^{\prime} \in S^{\prime}$ and $g \in B_{S^{\prime}}\left(R_{0}^{\kappa^{10}}\right)$, and furthermore $\left(\left(G^{\prime}\right)^{2},\left(S^{\prime}\right)^{(2)}\right)$ is a $\left(R_{0}^{1-2 \kappa^{10}}, d /\left(1-2 \kappa^{10}\right)\right)$-growth group. From Lemma 9.1 we conclude that

$$
U_{e}=I+\mathcal{O}_{\lambda}^{\lambda}\left(D^{O(1)} \varepsilon^{2}\right)+\mathcal{O}_{\lambda}\left(D^{O(1)} R_{0}^{-99 d}\right)
$$

for all $e \in\left(S^{\prime}\right)^{(2)}$, where the $O(1)$ exponents are larger than those in (54) by a multiplicative absolute constant.

Let $l$ be the first integer such that

$$
\varepsilon^{2^{l}}<R_{0}^{-100 d}
$$

or equivalently

$$
2^{l}\left(\kappa / 2 D^{2}\right)>100 d
$$

thus by (37), (45)

$$
l \ll \frac{d^{3}}{\kappa^{2}}(1+\log |S|) \ll \kappa^{-6} .
$$

We can iterate the above procedure $l$ times and conclude that the $l^{\text {th }}$ group $\left(G^{\prime}\right)^{(l)}$ in the derived series of $G^{\prime}$ is generated by a set $\left(S^{\prime}\right)^{(l)} \in B_{S^{\prime}}\left(R_{0}^{\kappa}\right)$ with the property that

$$
U_{e}=I+\mathcal{O}_{\lambda}^{\lambda}\left(D^{2^{O(l)}} \varepsilon^{2^{l}}\right)+\mathcal{O}_{\lambda}\left(D^{2^{O(l)}} R_{0}^{-99 d}\right)
$$

for all $e \in\left(S^{\prime}\right)^{(l)}$. By (47), (46), (40), (55) we may clean this up as

$$
U_{e}=I+\mathcal{O}_{\lambda}\left(R_{0}^{-90 d}\right)
$$

(say). From (41), (42) we conclude that

$$
\left\|\rho(e)\left(\lambda_{i} e_{i}\right)-\lambda_{i} e_{i}\right\|_{\ell^{2}\left(B_{S}\left(R_{1}\right)\right)} \ll R_{0}^{-80 d}
$$

(say) for all $1 \leq i \leq D$ and $e \in\left(S^{\prime}\right)^{(l)}$, which by (38) implies that

$$
\|\rho(e) f-f\|_{\ell^{2}\left(B_{S}\left(R_{1}\right)\right)} \ll R_{0}^{-70 d}
$$

(say) for all $f \in \Omega$ and $e \in\left(S^{\prime}\right)^{(l)}$. In particular, for any $R_{0}^{-K}$-harmonic Lipschitz function $u$, we see from the triangle inequality that

$$
\|\rho(e) u-u\|_{\ell^{2}\left(B_{S}\left(R_{1}\right)\right)} \ll R_{0}^{-60 d}
$$

(say) for all $e \in\left(S^{\prime}\right)^{(l)}$. Unpacking the definition of the $\overline{\ell^{2}\left(B_{S}\left(R_{1}\right)\right)}$ norm, and noting that $B_{S}\left(R_{1}\right)$ has cardinality at most $R_{0}^{d}$, we conclude that

$$
u(e g)-u(e h)=u(g)-u(h)+O\left(R_{0}^{-50 d}\right)
$$

(say) for all $g, h \in B_{S}\left(R_{1}\right)$ and $e \in\left(S^{\prime}\right)^{(l)}$ (here we use the symmetry of $\left.\left(S^{\prime}\right)^{(l)}\right)$. In particular, for $e, e^{\prime} \in\left(S^{\prime}\right)^{(l)}$ and $g \in B_{S}\left(R_{1} / 2\right)$ we have

$$
u\left(e e^{\prime} g\right)=u(e g)+u\left(e^{\prime} g\right)-u(g)+O\left(R_{0}^{-50 d}\right) .
$$

Reversing $e$ and $e^{\prime}$ and subtracting we conclude that

$$
u\left(e e^{\prime} g\right)=u\left(e^{\prime} e g\right)+O\left(R_{0}^{-50 d}\right),
$$

and thus

$$
u\left(\left[e, e^{\prime}\right] g\right)=u(g)+O\left(R_{0}^{-50 d}\right),
$$


for $e, e^{\prime} \in\left(S^{\prime}\right)^{(l)}$ and $g \in B_{S}\left(R_{1} / 4\right)$, which implies (after replacing $g$ by $g^{-1} h$, and $u(\cdot)$ by $u(g \cdot)$ that

$$
u\left(g\left[e, e^{\prime}\right] g^{-1} h\right)=u(h)+O\left(R_{0}^{-50 d}\right)
$$

for all $g, h \in B_{S}\left(R_{1} / 8\right)$ and $e, e^{\prime} \in\left(S^{\prime}\right)^{(l)}$.

By invoking Lemma 4.7 one last time, one can find a set $\left(S^{\prime}\right)^{(l+1)}$ of generators of $\left(G^{\prime}\right)^{(l+1)}$ in $B_{S^{\prime}}\left(R_{0}^{2 \kappa}\right)$ of the form $g\left[e, e^{\prime}\right] g^{-1}$ for some $e, e^{\prime} \in\left(S^{\prime}\right)^{(l)}$ and $g \in B_{S}\left(R_{0}^{2 \kappa}\right)$, and so from (57) we have concluded the following:

Theorem 9.3 (Many trivial directions for harmonic Lipschitz functions). Let $0<\kappa<0.1, R_{0}, d \geq 1$, and let $G$ be a $\left(R_{0}, d\right)$-growth group. Assume the bounds (45), (47) for some sufficiently large $C$. Then there exists a $\left(R_{0}^{1-\kappa^{10}}, d /\left(1-\kappa^{10}\right)\right)$ growth subgroup $\left(G^{\prime}, S^{\prime}\right)$ of $G$ of index at most $R_{0}^{\kappa^{10}}$ and a positive integer $l=O\left(\kappa^{-6}\right)$ such that $\left(G^{\prime}\right)^{(l+1)}$ is generated by a set $\left(S^{\prime}\right)^{(l+1)} \subset B_{S^{\prime}}\left(R_{0}^{2 \kappa}\right)$ obeying the bound

$$
u(e x)=u(x)+O\left(R_{0}^{-50 d}\right)
$$

for all $e \in\left(S^{\prime}\right)^{(l+1)}, x \in B_{S}\left(R_{0}^{1-3 \kappa}\right)$, and all $R_{0}^{-K}$-harmonic Lipschitz functions $u: G \rightarrow \mathbf{R}$, where $K$ is defined by $(30)$.

REMARK 9.4. An instructive example here is that of the Heisenberg group $G=$ $\left(\begin{array}{lll}1 & \mathbf{Z} & \mathbf{Z} \\ 0 & 1 & \mathbf{Z} \\ 0 & 0 & 1\end{array}\right)$, with the elementary row operations as generators. One can show that the only harmonic Lipschitz functions $u: G \rightarrow \mathbf{R}$ are those functions which are affine-linear combinations of the near-diagonal coefficients $x, z$ of the group element $\left(\begin{array}{lll}1 & x & y \\ 0 & 1 & z \\ 0 & 0 & 1\end{array}\right)$, and in particular such functions are invariant with respect to the vertical element $e:=\left(\begin{array}{lll}1 & 0 & 1 \\ 0 & 1 & 0 \\ 0 & 0 & 1\end{array}\right)$. One way to see this is to use the harmonicity to observe the reproducing formula $u=u * \sigma^{(m)}$ for any $m$, where $\sigma^{(m)}$ are the random walk distributions defined in Proposition 6.3. As $e$ is central, one then has $\partial_{e} u=u * \partial_{e} \sigma^{(m)}$, where $\partial_{e} f(x):=f(e x)-f(x)$. But a computation shows that the total variation of $\partial_{e} \sigma^{(m)}$ is $O\left(1 / m^{2}\right)$ (the intuition here is that $\sigma^{(m)}$ behaves like uniform probability measure on a box of dimensions $O(\sqrt{m})$ in the $x, z$ directions and $O(m)$ in the $y$ directions); in contrast, $u$, being Lipschitz, can only fluctuate by at most $O(m)$ on the bulk of the support of $\sigma^{(m)}$. Estimating things carefully and taking limits as $m \rightarrow \infty$ we conclude that $\partial_{e} u=0$, at which point it is easy to verify the claim. This example illustrates the general phenomenon, established in our companion paper [ShT] (using a different method), that Lipschitz harmonic functions on nilpotent groups vanish along iterated commutator directions; in fact modulo the constants they are exactly the additive group characters.

\section{Final Step for Proposition 5.2: Non-Trivial Harmonic Lipschitz Functions Have Large Range}

An easy application of the maximum principle shows that any non-constant harmonic function $u: G \rightarrow \mathbf{R}$ must attain an infinite number of values. We thus expect any $\varepsilon$-harmonic Lipschitz function $u: G \rightarrow \mathbf{R}$ obeying some non-degeneracy 
condition (e.g. a lower bound on $\nabla u(\mathrm{id}))$ to also take on a large number of values in any given ball $B_{S}(R)$.

In fact we will need a stronger result (under a polynomial growth hypothesis), which asserts that a non-degenerate $\varepsilon$-harmonic function must in fact fluctuate by $\gg R$ on the ball $B_{S}(R)$ :

Proposition 10.1 (Lower bound on range). Let $(G, S)$ be a $\left(R_{0}, d\right)$-growth group for some $R_{0}, d \geq 1$, let $0<\kappa<0.1$, and suppose that (12) holds for some sufficiently large absolute constant $C$. Let $u: G \rightarrow \mathbf{R}$ be an $R_{0}^{-K}$-harmonic function such that $|\nabla u(\mathrm{id})| \geq 1 /|S|$, where $K$ was defined in (12). Then for every $R_{0}^{1-5 \kappa} \leq R \leq R_{0}^{1-2 \kappa}$ we have

$$
\sup _{x \in B_{S}(R)}|u(x)-u(\mathrm{id})| \gg O(|S|)^{-O\left(d^{3} / \kappa^{2}\right)} R_{0}^{-\kappa} R .
$$

Proof. Our main tool here will be the quantitative Kleiner theorem (Theorem 7.1). This theorem gives us a space $V \subset \mathbf{R}^{G}$ of dimension

$$
D:=\operatorname{dim}(V)=O(|S|)^{O\left(d^{3} / \kappa^{2}\right)}
$$

such that for every $g \in G$, there exists $v_{g} \in V$ such that

$$
\left\|\rho(g) u-v_{g}\right\|_{\ell^{2}\left(B_{S}\left(R_{0}^{1-\kappa}\right)\right)} \leq R_{0}^{-100 d},
$$

and in particular

$$
\left\|\rho(g) \nabla u-\nabla v_{g}\right\|_{\ell^{2}\left(B_{S}\left(R_{0}^{1-\kappa}\right)\right)} \leq|S| R_{0}^{-100 d} .
$$

The balls $B_{S}\left(R^{\prime}\right)$ for $R_{0}^{-\kappa} R \leq R^{\prime} \leq R$ are increasing in $R^{\prime}$, and have cardinality between 1 and $R_{0}^{d}$. By the pigeonhole principle, one may thus find a radius $2 R_{0}^{-\kappa} R \leq$ $R^{\prime} \leq R$ such that

$$
\left|B_{S}\left(R^{\prime}\right)\right| \ll O(1)^{d / \kappa}\left|B_{S}\left(R^{\prime} / 10\right)\right| \text {. }
$$

Fix this $R^{\prime}$. By subtracting off a constant, we may assume $u($ id $)=0$. Write $\alpha:=R^{\prime} R_{0}^{-K}+R^{-1} \sup _{x \in B_{S}\left(R^{\prime}\right)}|u(x)|$, then

$$
\sum_{x \in B_{S}\left(R^{\prime}\right)}|u(x)|^{2} \leq\left(R^{\prime}\right)^{2} \alpha^{2}\left|B_{S}\left(R^{\prime}\right)\right| .
$$

Applying Proposition 7.3 we conclude that

$$
\|\nabla u\|_{\ell^{2}\left(B_{S}\left(R^{\prime} / 2\right)\right)} \ll|S|^{O(1)} \alpha\left|B_{S}\left(R^{\prime}\right)\right|^{1 / 2} .
$$

Now, let $g_{1}, \ldots, g_{D+1}$ be chosen uniformly at random from $B_{S}\left(R^{\prime} / 10\right)$. From (61), (62), and Chebyshev's inequality we see that for each distinct $1 \leq i, j \leq D+1$, we have

$$
\left|\rho\left(g_{i}\right) \nabla(u)\left(g_{j}\right)\right| \leq \frac{1}{100|S| D}
$$

with probability at least $1-O(|S|)^{O\left(d^{3} / \kappa^{2}\right)} \alpha^{2}$. By the union bound, we thus have (63) for all distinct $1 \leq i, j \leq D+1$ with probability at least $1-O(|S|)^{O\left(d^{3} / \kappa^{2}\right)} \alpha^{2}$. Meanwhile, for $i=j$, we have

$$
\left|\rho\left(g_{i}\right) \nabla(u)\left(g_{j}\right)\right| \geq \frac{1}{|S|}
$$


by hypothesis. Applying (63) we see that

for $i \neq j$, and

$$
\left|\nabla\left(v_{g_{i}}\right)\left(g_{j}\right)\right| \leq \frac{1}{200|S| D}
$$

$$
\left|\nabla\left(v_{g_{i}}\right)\left(g_{j}\right)\right| \geq \frac{1}{2|S|}
$$

for $i=j$. The matrix $\left(\nabla\left(v_{g_{i}}\right)\left(g_{j}\right)\right)_{1 \leq i, j \leq D+1}$ is then diagonally dominant and thus invertible; however, the $v_{g_{1}}, \ldots, v_{g_{D+1}}$ lie in a $D$-dimensional space and thus must have a linear dependence. This leads to a contradiction unless the stated event occurs with zero probability; this forces $\alpha \gg O(|S|)^{-O\left(d^{3} / \kappa^{2}\right)}$, and the claim follows.

We can combine this with Theorem 9.3 and Proposition 6.3 to obtain a crucial reduction in growth order, from $d$ to approximately $d-1$ :

Corollary 10.2 (Reduction in growth order). Let $0<\kappa<0.1, R_{0}, d \geq 1$, and let $(G, S)$ be a $\left(R_{0}, d\right)$-growth group. Assume the bounds (45), (47) for some sufficiently large $C$. Then at least one of the following holds:

- $G=B_{S}\left(R_{0}^{\exp \left(\exp \left(\kappa^{-O(1)}\right)\right)}\right)$.

- There exists a $\left(R_{0}^{1-\kappa^{10}}, d /\left(1-\kappa^{10}\right)\right)$-growth subgroup $\left(G^{\prime}, S^{\prime}\right)$ of $G$ of index at most $R_{0}^{\kappa^{10}}$ and a positive integer $l=O\left(\kappa^{-6}\right)$ such that $\left(G^{\prime}\right)^{(l+1)}$ is generated by a set $\left(S^{\prime}\right)^{(l+1)} \subset B_{S^{\prime}}\left(R_{0}^{2 \kappa}\right)$ for which $\left(\left(G^{\prime}\right)^{(l+1)},\left(S^{\prime}\right)^{(l+1)}\right)$ is a $\left(R_{0}^{1-4 \kappa},(d-1+6 \kappa) /(1-4 \kappa)\right)$-growth group.

Proof. Applying Proposition 6.3 we see that either the first conclusion holds, or there exists a $R_{0}^{-K}$-harmonic Lipschitz function with $|\nabla u(\mathrm{id})| \geq 1 /|S|$, where $K$ is defined by (12). Applying Theorem 9.3 we can ensure that (58) holds for this value of $u$; iterating this we see in particular that

$$
u(g x)=u(x)+O\left(R_{0}^{-40 d}\right)
$$

for all $e \in B_{\left(S^{\prime}\right)^{(l+1)}}\left(R_{0}^{1-4 \kappa}\right), x \in B_{S}\left(R_{0}^{1-4 \kappa}\right)$.

By Proposition 10.1 (and cleaning up the constants) we have

$$
\sup _{x \in B_{S}\left(R_{0}^{1-4 \kappa}\right)}|u(x)-u(\mathrm{id})| \geq 10 R_{0}^{1-6 \kappa}
$$

and thus there exists a path of length at most $R_{0}^{1-4 \kappa}$ starting at the origin on which $u$ fluctuates by at least $\geq 10 R_{0}^{1-6 \kappa}$. Since $u$ also has Lipschitz constant at least 1 , we can thus find $M>R_{0}^{1-6 \kappa}$ points $x_{1}, \ldots, x_{M} \in B_{S}\left(R_{0}^{1-4 \kappa}\right)$ such that $u\left(x_{1}\right), \ldots, u\left(x_{M}\right)$ all differ by at least 1 . Combining this with (64) we see that the sets $B_{\left(S^{\prime}\right)(l+1)}\left(R_{0}^{1-4 \kappa}\right) \cdot x_{m}$ for $1 \leq m \leq M$ are disjoint. But these sets all lie in $B_{S}\left(R_{0}\right)$, which has cardinality at most $R_{0}^{d}$. We conclude that

$$
B_{\left(S^{\prime}\right)^{(l+1)}}\left(R_{0}^{1-4 \kappa}\right) \leq R_{0}^{d} / M=R_{0}^{d-1+6 \kappa}
$$

and the claim follows.

Finally, we can prove Proposition 5.2. 
Proof of Proposition 5.2. Applying Lemma 4.2 with $\kappa=c / d$ for some sufficiently small absolute constant $c>0$, one can find a $\left(R_{0}^{c / d}, R_{0}^{c / d}\right)$-subgroup $(\tilde{G}, \tilde{S})$ of $G$ which is a $\left(R_{0}^{1-\frac{1}{100 d}}, d-0.01\right)$-growth group and with $|\tilde{S}| \ll O(1)^{d^{2}}$ and $G=\tilde{G} \cdot B_{S}\left(R_{0}^{\frac{1}{100 d}}\right)$. We then apply Corollary 10.2 to $(\tilde{G}, \tilde{S})$ with $\kappa:=c / d^{2}$ for some sufficiently small absolute constant $c>0$. If the first conclusion of Corollary 10.2 holds, then the properties relating $(G, S)$ to $(\tilde{G}, \tilde{S})$ imply that $G=B_{S}\left(R_{0}^{\exp \left(\exp \left(O(d)^{O(1)}\right)\right)}\right)$. If the second conclusion of Corollary 10.2 holds, then the claim follows after substituting in the value of $\kappa$ (and replacing $l$ by $l+1$ ), and using Lemma 4.1. (Observe from Lemma 4.8 that $G^{\prime}$ is a $\left(R_{0}^{\kappa^{10}}, R_{0}^{\kappa^{10}}, 1\right)$-subgroup of $G$.)

\section{First Step for Proposition 5.3: Reduction to a Cyclic Base}

We now begin the proof of Proposition 5.3. In this section we execute the first step of this proof, which is to reduce to the case when the base group $L$ is cyclic rather than solvable.

By replacing $S$ with $S \cup S_{H}$, replacing $R_{0}$ by $R_{0}^{9 / 10}$, and adjusting $d$ and $C$ appropriately, we may assume that $S_{H} \subset S$ in Proposition 5.3, thus $\left(H, S_{H}\right)$ is now a $(\infty, 1)$-subgroup of $(G, S)$.

We now claim that Proposition 5.3 follows from the $l=1$ case of this proposition, and specifically from

Proposition 11.1 (Semi-quantitative Milnor-Wolf theorem, first reduction). Let $r, R_{0}, d \geq 1$, and suppose that

$$
R_{0} \geq C^{d} r^{C}
$$

for some sufficiently large absolute constant $C$. Suppose we have a short exact sequence

$$
0 \rightarrow H \rightarrow G \rightarrow A
$$

of groups, where $G=(G, S)$ is a $\left(R_{0}, d\right)$-growth group, $\left(H, S_{H}\right)$ is a virtually $r$ nilpotent $(\infty, 1)$-subgroup of $(G, S)$, and $A$ is abelian. Then $(G, S)$ is virtually $r+O(1)^{d}$-nilpotent.

To see how Proposition 11.1 implies Proposition 5.3, we induct on $l$. The case $l=1$ already follows from Proposition 11.1, so suppose that $l \geq 2$ and the claim has already been proven for $l-1$.

Let $\kappa:=1 / 100 d l$, then by Lemma 4.7 we may find a set of generators $S^{\prime} \subset B_{S}\left(R_{0}^{\kappa}\right) \cap[G, G]$ for $[G, G]$. If we then set $\tilde{G}:=\langle[G, G], H\rangle$ and $\tilde{S}:=$ $S_{H} \cup S^{\prime} \subset B_{S}\left(R_{0}^{\kappa}\right)$, we see that $(\tilde{G}, \tilde{S})$ is a $\left(R_{0}^{1-\kappa}, d /(1-\kappa)\right)$-growth group, and we have the short exact sequence

$$
0 \rightarrow H \rightarrow \tilde{G} \rightarrow[N, N] \rightarrow 0 .
$$

Of course, $[N, N]$ is solvable of derived length at most $l-1$, so by induction hypothesis $(\tilde{G}, \tilde{S})$ is virtually $r+(l-1) O(1)^{d}$-nilpotent. (Note that even after iterating this induction hypothesis $l$ times, the order of growth $d$ of $\tilde{G}$ does not increase significantly, thanks to the choice of $\kappa$, so for the purposes of computing quantitative 
bounds one can treat $d$ as constant throughout this iteration.) On the other hand, $G / \tilde{G}$ is abelian. If we then apply Proposition 11.1 to the short exact sequence

$$
0 \rightarrow \tilde{G} \rightarrow G \rightarrow G / \tilde{G} \rightarrow 0
$$

we obtain the claim (for $C$ large enough).

Now suppose we are in the situation of Proposition 11.1. At present, the abelian group $\left(A, S_{A}\right)$ could have many generators; but we can cut down the number of generators to a quantity depending on $d$. Indeed, as $G=(G, S)$ is an $\left(R_{0}, d\right)$-growth group, we see that $\left(A, S_{A}\right)$ is also an $\left(R_{0}, d\right)$-growth group, where $S_{A}$ is the projection of $S$ to $A$. Applying Lemma 4.2 , we may find a $\left(R_{0}^{0.9}, d / 0.9\right)$-growth $\left(R_{0}^{0.1}, R_{0}^{0.1}\right)$ subgroup $\left(A^{\prime}, S_{A^{\prime}}\right)$ of $(A, S)$ with $\left|S_{A^{\prime}}\right| \ll O(1)^{d}$. Let $G^{\prime}$ be the preimage of $A^{\prime}$ in $G$, and let $S^{\prime}:=B_{S}\left(R_{0}^{0.1}\right) \cap A^{\prime}$, then $\left(G^{\prime}, S^{\prime}\right)$ is a $\left(R_{0}^{0.1}, R_{0}^{0.1}\right)$-subgroup of $(G, S)$. We may thus replace $G, A, S$ by $G^{\prime}, A^{\prime}, S^{\prime}$ in Proposition 11.1 (adjusting $d$ and $C$ slightly), thus allowing us to reduce to the case when $A$ is generated by at most $m=O(1)^{d}$ elements $a_{1}, \ldots, a_{m}$, and furthermore that we may assume that elements are contained in the projection of $S$ to $A$.

As $\left(H, S_{H}\right)$ is a $(\infty, 1)$-subgroup of $(G, S), S_{H}$ is contained in $S$. We can then discard all elements of $S$ other than those in $S_{H}$ and those that are projecting to $A$, and thus assume that $S$ takes the form

$$
S=S_{H} \cup\left\{e_{1}, \ldots, e_{m}, e_{1}^{-1}, \ldots, e_{m}^{-1}\right\}
$$

where $e_{1}, \ldots, e_{m} \in G$ projects to the generators $a_{1}, \ldots, a_{m}$ of $A$.

An easy induction on $m$ then allows us to reduce to the one-dimensional case $m=1$, and specifically from

Proposition 11.2 (Semi-quantitative Milnor-Wolf theorem, second reduction).

Let $r, R_{0}, d \geq 1$, and suppose that

$$
R_{0} \geq C^{d} r^{C}
$$

for some sufficiently large absolute constant $C$. Suppose we have a short exact sequence

$$
0 \rightarrow H \rightarrow G \rightarrow A
$$

of groups, where $(G, S)$ is a $\left(R_{0}, d\right)$-growth group, $\left(H, S_{H}\right)$ is a virtually $r$-nilpotent subgroup, and $A$ is cyclic. Suppose also that $S=S_{H} \cup\left\{e, e^{-1}\right\}$ for some $e \in G$. Then $G$ is virtually $r+1$-nilpotent.

It remains to establish Proposition 11.2. This will be accomplished in section 14, after some preliminaries in sections 12, 13 .

\section{Second Step for Proposition 5.3: Slow Growth of Iterated Conjugation}

Suppose we are in the situation of Proposition 11.2. The short exact sequence forces $H$ to be a normal subgroup of $G$, and so the generator $e$ induces an automorphism $T: H \rightarrow H$ defined by the conjugation operation $T h:=e h e^{-1}$. As $S_{H}$ generates $H$, 
we see that there exists some radius $R$ such that $T\left(S_{H}\right), T^{-1}\left(S_{H}\right) \subset B_{S_{H}}(R)$. This gives rise to the crude bound

$$
\left\|T^{n} h\right\|_{S_{H}} \leq R^{|n|}\|h\|_{S_{H}}
$$

for all $n \in \mathbf{Z}$ and $h \in H$, where the norms \|\|$_{S_{H}}$ were defined in Definition 1.1.

This bound is useless for our purposes because the argument gives no effective bound on $R$. However, it turns out that one can use the polynomial growth hypothesis $\left|B_{S}\left(R_{0}\right)\right| \leq R_{0}^{d}$ to obtain a much stronger bound, after adjusting the generating set $S$ slightly. Namely, we have

Proposition 12.1 (Slow growth). Let $R_{0}, d \geq 1$ be such that $R_{0} \geq C^{d}$ for some sufficiently large absolute constant $C$. Suppose we have a short exact sequence

$$
0 \rightarrow H \rightarrow G \rightarrow A
$$

of groups, where $A$ is cyclic. Suppose also that $G=(G, S)$ is a $\left(R_{0}, d\right)$-growth group with $S=S_{H} \cup\left\{e, e^{-1}\right\}$, where $S_{H} \subset H$ generates $H$, and the projection $e$ of $S$ to $A$ generates $A$. Then there exists a symmetric set $\tilde{S} \subset B_{S}\left(R_{0}^{1 / 10}\right) \cap H$ generating $H$ such that

$$
\left\|T^{n} h\right\|_{\tilde{S}} \ll \exp \left(|n| / R_{0}^{0.01}\right)\|h\|_{\tilde{S}}
$$

for all $n \in \mathbf{Z}$ and $h \in H$, where $T: H \rightarrow H$ is the conjugation map $T h:=e h e^{-1}$.

Proof. By hypothesis, $\left|B_{S}\left(R_{0}\right)\right| \leq R_{0}^{d}$. From the pigeonhole principle and the lower bound on $R_{0}$, we may then find a radius $R_{0}^{1 / 30} \leq R \leq R_{0}^{1 / 20}$ such that

$$
\left|B_{S}(10 R) \cap H\right| \leq O(1)^{d}\left|B_{S}(R) \cap H\right| .
$$

Fix this $R$. For every $0 \leq N \leq R$, consider the sets

$$
A_{N}:=\bigcup_{-N \leq n \leq N} T^{n}\left(B_{S}(R) \cap H\right) .
$$

Observe that the $A_{N}$ are symmetric subsets of $H$ that are increasing in $N$, and that $B_{S}(R) \cap H \subset A_{N} \subset A_{N} \cdot\left(B_{S}(R) \cap H\right) \subset B_{S}(10 R) \cap H$, and thus

$$
\left|B_{S}(R) \cap H\right| \leq\left|A_{N} \cdot\left(B_{S}(R) \cap H\right)\right| \leq O(1)^{d}\left|B_{S}(R) \cap H\right| .
$$

By another application of the pigeonhole principle, there exists $0 \leq N \leq R / 2$ such that

$$
\left|A_{N+C^{-d} R} \cdot\left(B_{S}(R) \cap H\right)\right|<\left|A_{N} \cdot\left(B_{S}(R) \cap H\right)\right|+\left|B_{S}(R) \cap H\right|
$$

for some large absolute constant $C$.

Fix this $R$, and set $\tilde{S}:=A_{N}$, thus $\tilde{S}$ is a symmetric subset of $B_{S}\left(R_{0}^{1 / 10}\right) \cap H$ which contains $S_{H}$ and thus generates $H$. For every $-C^{-d} R \leq n \leq C^{-d} R$, we see from construction that

$$
\left|\left(\tilde{S} \cup T^{n} \tilde{S}\right) \cdot\left(B_{S}(R) \cap H\right)\right|<\left|\tilde{S} \cdot\left(B_{S}(R) \cap H\right)\right|+\left|B_{S}(R) \cap H\right| .
$$

This implies that

$$
\tilde{S} \cup T^{n} \tilde{S} \subset \tilde{S} \cdot\left(B_{S}(R) \cap H\right) \cdot\left(B_{S}(R) \cap H\right)^{-1},
$$

for if there was an element $x \in \tilde{S} \cup T^{n} \tilde{S}$ which did not lie in $\tilde{S} \cdot\left(B_{S}(R) \cap H\right) \cdot\left(B_{S}(R) \cap H\right)^{-1}$, then the set $\left.x \cdot B_{S}(R) \cap H\right)$ would lie in $\left(\tilde{S} \cup T^{n} \tilde{S}\right) \cdot\left(B_{S}(R) \cap H\right)$ but be disjoint from 
$\tilde{S} \cdot\left(B_{S}(R) \cap H\right)$, contradicting (65). Since $B_{S}(R) \cap H$ is contained in the symmetric set $\tilde{S}$, we conclude that

$$
T^{n} \tilde{S} \subset B_{\tilde{S}}(3)
$$

and thus (as $T^{n}: H \rightarrow H$ is an automorphism)

$$
\left\|T^{n} h\right\|_{\tilde{S}} \leq 3\|h\|_{\tilde{S}}
$$

for all $h \in H$ and $-C^{-d} R \leq n \leq C^{-d} R$. From iteration we then obtain

$$
\left\|T^{n} h\right\|_{\tilde{S}} \ll \exp \left(C^{d}|n| / R\right)\|h\|_{\tilde{S}}
$$

for all $h \in H$ and $n \in \mathbf{Z}$, and the claim then follows from the lower bounds on $R$ and $R_{0}$.

\section{Third Step for Proposition 5.3: The Case of an Action on Lattices}

We are still preparing for the proof of Proposition 11.2 (and hence Proposition 5.3). Proposition 12.1 places us in the setting of an automorphism $T: H \rightarrow H$ on some virtually nilpotent group $H$ whose iterates grow very slowly with respect to some word norm \|\|$_{S^{\prime}}$. In this section we study a key model case of this situation, in which $H=\mathbf{Z}^{D}=\left(\mathbf{Z}^{D},+\right)$ is a lattice of bounded dimension, and the word norm \|\|$_{S^{\prime}}$ is replaced by the Euclidean norm $|\cdot|$ on $\mathbf{R}^{D}$ (and hence $\mathbf{Z}^{D}$ ). The study of this case is central to all arguments of Milnor-Wolf type, see e.g. [W], or the appendix by Tits in $[\mathrm{Gr}]$. The main result to establish in this section is as follows.

Proposition 13.1 (Dichotomy between periodicity and exponential growth). Let $D \geq 1$, and let $T \in S L_{D}(\mathbf{Z})$ be an invertible linear transformation $T: \mathbf{Z}^{D} \rightarrow \mathbf{Z}^{D}$. Then at least one of the following statements holds:

- (Periodicity) There exists a non-zero vector $w \in \mathbf{Z}^{D}$ and an integer $1 \leq n \leq$ $D^{O(1)}$ such that $T^{n} w=w$.

- (Growth) For any $N \geq 1$, there exists a non-zero vector $v=v_{N} \in \mathbf{Z}^{D}$ such that

$$
\left|T^{N} v\right| \gg \exp \left(c N / D^{O(1)}\right)|v|
$$

for some absolute constant $c>0$.

Proof. Let $\lambda_{1}, \ldots, \lambda_{D} \in \mathbf{C}$ be the eigenvalues of $T$ (counting multiplicity). Then $\lambda_{1} \ldots \lambda_{D}=\operatorname{det}(T)= \pm 1$, which implies that $\max _{1 \leq j \leq D}\left|\lambda_{j}\right| \geq 1$.

Suppose first that $\max _{1 \leq j \leq D}\left|\lambda_{j}\right|=1$, then all the $\lambda_{j}$ are algebraic integers whose Galois conjugates all lie on the unit circle. By a classical result of Kronecker[Kr] (or Remark 13.2 below), this implies that the $\lambda_{j}$ are all roots of unity. If one of the $\lambda_{j}$ is a primitive $n^{\text {th }}$ root of unity, then the degree $\phi(n)$ of that root cannot exceed $D$. Elementary number theory (using the prime factorization of $n$ ) yields that $\phi(n) \geq c_{\varepsilon} n^{1-\varepsilon}$ for any $\varepsilon>0$ and some constant $c_{\varepsilon}>0$, and thus we have $n=O\left(D^{O(1)}\right)$. Then $T^{n}-I$ has non-trivial kernel, and we obtain the periodicity claim. 
Now suppose instead that $\max _{1<j<D}\left|\lambda_{j}\right|>1$. Applying a result of Dobrowolski [D], we conclude in fact that $\max _{1 \leq j \leq D}\left|\lambda_{j}\right|>1+c D^{-O(1)}$ for some $c>0$ (in fact the more precise bound $1+c \frac{\log ^{3} D}{D \log \log ^{3} D}$ is known). In particular we may assume that $\left|\lambda_{1}\right| \geq 1+c D^{-O(1)}$. Let $v_{1}$ be an eigenvector of $\lambda_{1}$, then clearly

$$
\left|T^{N} v_{1}\right| \gg \exp \left(O(1)^{-D} N\right)\left|v_{1}\right| \text {. }
$$

From the triangle inequality we see that either the real or complex part of $v_{1}$ obeys a similar growth bound. Approximating this real or complex part by a non-zero vector with rational coefficients and then clearing denominators, we obtain the claim.

REMARK 13.2. For our applications, one may replace Dobrowolski's lower bound of $1+c D^{-O(1)}$ here with the more elementary bound of $1+\exp (-O(D))$, the proof of which we sketch as follows. Suppose we had an algebraic integer $\lambda$ of degree $D$, all of whose Galois conjugates were at most $1+C^{-D}$ for some large absolute constant $C$, but which was not a root of unity. Using the minimal polynomial of $\lambda$, one can find a diagonalizable transformation $T: \mathbf{C}^{D^{\prime}} \rightarrow \mathbf{C}^{D^{\prime}}$ for some $D^{\prime} \leq D$ with integer coefficients and with eigenvalues equal to these Galois conjugates. One can then use the corresponding eigenvectors to design a symmetric convex body $B$ with the property that $T^{n}(B) \subset 2 \cdot B$ for all $n \leq(C / 10)^{D}$. By rescaling, one may assume that $B$ contains a lattice vector $v$ in $\mathbf{Z}^{D}$ on its boundary, but no non-zero lattice vector in its interior. As $\lambda$ is not a root of unity, the images of $T^{n} v$ are all distinct, and so $2 \cdot B$ contains at least $(C / 10)^{D}$ lattice vectors; but standard volume packing arguments show that this cannot be the case for $C$ large enough.

\section{Final Step for Proposition 5.3: Eliminating the Finite Factors}

We are now ready to prove Proposition 11.2. Let $r, R_{0}, d, H, G, A, S, S_{H}, e$ be as in Proposition 11.2.

By hypothesis, $H$ contains a finite-index nilpotent subgroup $H^{\prime}$ of Hirsch length at most $r$, and thus step $s \leq r$. As is well known, $H^{\prime}$ then contains a finite-index subgroup $H^{\prime \prime}$ which is torsion free.

The group $H^{\prime \prime}$ need not be $T$-invariant. However, observe from Legendre's theorem that $h^{\left|H: H^{\prime \prime}\right|} \in H^{\prime \prime}$ for all $h \in H$. Thus, if we let $H^{\prime \prime \prime}$ be the group generated by $\left\{h^{\left|H: H^{\prime \prime}\right|}: h \in H\right\}$, then $H^{\prime \prime \prime}$ is a $T$-invariant normal subgroup of $H^{\prime \prime}$. The nilpotent quotient group $H^{\prime \prime} / H^{\prime \prime \prime}$ is generated by finitely many torsion elements, hence it is finite. We conclude that $H^{\prime \prime \prime}$ is a $T$-invariant finite-index torsion-free subgroup of $H$, and thus has Hirsch length $r$. Let $H_{i}^{\prime \prime \prime}$ be an upper central series for $H^{\prime \prime \prime}$ terminating at $H_{s}^{\prime \prime \prime}:=H^{\prime \prime \prime}$. By torsion freeness, we can identify $H_{i}^{\prime \prime \prime} / H_{i-1}^{\prime \prime \prime}$ with $\mathbf{Z}^{d_{i}}$ for all $i \leq s$, with $\Sigma d_{i}=r$.

Meanwhile, by Proposition 12.1, there exists a set $\tilde{S} \subset B_{S}\left(R_{0}^{1 / 10}\right) \cap H$ generating $H$ such that

$$
\left\|T^{n} h\right\|_{\tilde{S}} \ll \exp \left(|n| / R_{0}^{0.01}\right)\|h\|_{\tilde{S}}
$$

for all $n \in \mathbf{Z}$ and $h \in H$, where $T: H \rightarrow H$ is the conjugation map $T h:=e h e^{-1}$. 
We claim that the $\tilde{S}$ norm and $S^{\prime \prime \prime}$ norm are comparable on $H^{\prime \prime \prime}$, i.e. there exists an $M>0$ such that

$$
M^{-1}\|h\|_{S^{\prime \prime \prime}} \leq\|h\|_{\tilde{S}} \leq M\|h\|_{S^{\prime \prime \prime}}
$$

for all $h \in H^{\prime \prime \prime}$. (We do not claim an effective bound on $M$.) The second inequality follows for sufficiently large $M$ since each element of $S^{\prime \prime \prime}$ is generated by $\tilde{S}$. To get the former inequality, observe as in Remark 1.4 that we can partition $H$ into finitely many cosets $x_{1} \cdot H^{\prime \prime \prime}, \ldots, x_{m} \cdot H^{\prime \prime \prime}$ (with $x_{1}=\mathrm{id}$, say) with relations $e x_{i}=x_{j_{e, i}} g_{e, i}$ for all $1 \leq i \leq m, e \in \tilde{S}$ and some $1 \leq j_{e, i} \leq m, g_{e, i} \in H^{\prime \prime \prime}$; iterating these relations we see that any product of $R$ elements of $\tilde{S}$ can be expressed as the product of one of the $x_{i}$ times $R$ of the $g_{e, i}$; in particular, any product of $R$ elements of $\tilde{S}$ that lie in $H^{\prime \prime \prime}$ can be expressed as the product of $R$ of the $g_{e, i}$, giving the desired inequality for some $M$.

Inserting (68) into (67) we conclude that

$$
\left\|T^{n} h\right\|_{S^{\prime \prime \prime}} \ll M^{2} \exp \left(|n| / R_{0}^{0.01}\right)\|h\|_{S^{\prime \prime \prime}}
$$

for all $h \in H^{\prime \prime \prime}$ and $n \in \mathbf{Z}$.

We shall now argue similarly to Tits in his appendix to Gromov's [Gr], but in a quantitative manner. The automorphism $T$ preserves each $H_{i}^{\prime \prime \prime}$ and thus acts on the successive abelian quotients which are torsion free as well (cf. [M1]): $H_{i}^{\prime \prime \prime} / H_{i-1}^{\prime \prime \prime} \cong \mathbf{Z}^{d_{i}}$. Beginning from the top, by Proposition 13.1, either $H_{s}^{\prime \prime \prime} / H_{s-1}^{\prime \prime \prime} \cong \mathbf{Z}^{d_{s}}$ contains a nonzero periodic vector with period at most $O\left(d_{s}\right)^{O(1)}=O(r)^{O(1)}$, or else one can find for any $n \geq 1$ a non-zero vector $v$ in $\mathbf{Z}^{d_{s}}$ such that

$$
\left|T^{n} v\right| \gg \exp \left(n / O(r)^{O(1)}\right)|v| \text {. }
$$

But by the lower bound on $R_{0}$, the latter possibility contradicts (69) if one takes $n$ sufficiently large, since one can use the norm in $\mathbf{Z}^{d_{s}}$ as a lower bound for the $S^{\prime \prime \prime}$ norm. Thus $\mathbf{Z}^{d_{s}}$ contains a non-zero periodic vector $v$ with some period $1 \leq p_{0} \ll$ ${ }_{r} O(1)$. This implies that the operator $T^{p_{0}}-I$ (viewed as a linear transformation on $\mathbf{Z}^{d_{s}}$ ) maps $\mathbf{Z}^{d_{s}}$ to a $T$-invariant subgroup of $\mathbf{Z}^{d_{s}}$ of infinite index. Hence the map $\Phi_{p_{0}}: h \mapsto\left(T^{p_{0}} h\right) h^{-1}$ maps $H_{(0)}^{\prime \prime \prime}:=H_{s}^{\prime \prime \prime} / H_{s-1}^{\prime \prime \prime}$ into a $T$-invariant subgroup of it, $H_{(1)}^{\prime \prime \prime}$ (of strictly smaller dimension). We can iterate this process, finding a $1 \leq p_{1} \leq O(r)^{O(1)}$ such that $\Phi_{p_{1}}$ maps $H_{(1)}^{\prime \prime \prime}$ into a $T$-invariant subgroup $H_{(2)}^{\prime \prime \prime}$ of strictly smaller dimension, until these groups vanish, i.e. $T$ takes $H_{s}^{\prime \prime \prime}=H^{\prime \prime \prime}$ into $H_{s-1}^{\prime \prime \prime}$. But then we can work on the abelian quotient $H_{s-1}^{\prime \prime \prime} / H_{s-2}^{\prime \prime \prime} \cong \mathbf{Z}^{d_{s-1}}$ instead. Standard nilpotent algebra then tells us that the square root ||$^{1 / 2}$ of the norm on $\mathbf{Z}^{d_{s-1}}$ is essentially a lower bound for the $S^{\prime \prime \prime}$ norm (continuing to rely heavily on torsion freeness), and we can continue the argument much as before, reducing the dimension of the $T$-invariant subgroups until they fall into $H_{s-2}^{\prime \prime \prime}$, then $H_{s-3}^{\prime \prime \prime}$, etc., eventually collapsing to the identity. Since $H^{\prime \prime \prime}$ had Hirsch length $r$, we can thus find $1 \leq p_{1}, \ldots, p_{m} \ll r^{O(1)}$ for some $1 \leq m \leq r$ and $T$-invariant subgroups

$$
H^{\prime \prime \prime}=H_{(1)}^{\prime \prime \prime} \geq H_{(2)}^{\prime \prime \prime} \geq \ldots \geq H_{(m+1)}^{\prime \prime \prime}=\{\mathrm{id}\}
$$

such that $\Phi_{p_{i}}$ maps $H_{(i)}^{\prime \prime \prime}$ to $H_{(i+1)}^{\prime \prime \prime}$ for all $1 \leq i \leq m$. If we let $P:=p_{1} \ldots p_{m} \ll r^{O}(r)$ be the product of all these periods, it follows that $T^{P}$ acts unipotently on $H^{\prime \prime \prime}$. This 
implies that

$$
\left\{e^{n P} h: n \in \mathbf{Z}, h \in H^{\prime \prime \prime}\right\}
$$

is a finite-index subgroup of $G$ which is nilpotent of Hirsch length (and thus step) at most $r+1$, and Proposition 11.2 follows.

\section{Effectivization}

In this section we discuss some fully effective results including Theorem 1.9 and the case of torsion-free groups, and mainly remark on the modifications needed in the above arguments to make the bound on the quantity $K\left(R_{0}, d\right)$ in Theorem 1.8 effective. However, we will not provide complete details here for the latter, as they are rather lengthy, and the final bound on $K\left(R_{0}, d\right)$ obtained by this process is quite poor.

15.1 Some fully quantitative results. We begin by mentioning one quantitative geometric application of Theorem 1.8, based on Milnor's result [Mi2] as used in [Gr, p. 72].

Corollary 15.2 (to Theorem 1.8). Let $(V, d)$ be a complete Riemannian manifold of dimension $n$, and $K \geq 0$ be such that the values of the Ricci tensor on the unit tangent bundle at all points is bounded from below by $-(n-1) K$. Let $\Gamma$ be a group of isometries of $V$ generated by the finite subset $S$. For a point $v \in V$, define

$$
\delta_{v}:=\inf \{d(\gamma v, v) \mid \gamma \in \Gamma\} \quad \Delta_{v}:=\sup \{d(\gamma v, v) \mid \gamma \in S\} .
$$

If for some $v \in V$ and some $R>\exp \left(\exp \left(C(2 n)^{C}\right)\right.$ the inequality,

$$
4 \frac{\Delta_{v}}{\delta_{v}} \exp \left(2 \pi \Delta_{v} \sqrt{K} R\right)<R
$$

holds, where $C$ is the absolute constant of Theorem 1.8, then $\Gamma$ is virtually nilpotent.

This follows immediately from Milnor's inequality[Mi2]

$$
\left|B_{S}(R)\right| \leq 4^{n}\left(\frac{\Delta_{v}}{\delta_{v}}\right)^{n} R^{n} \exp \left(2 \pi \Delta_{v} \sqrt{K} R\right)
$$

combined with the main Theorem 1.8, to which the condition in Corollary 15.2 is tailored with the value $d=2 n$. Of course, the corollary is of interest in situations where $K$, or both $\Delta_{v}, \delta_{v}$ and their quotient, are small.

We next discuss Theorem 1.9. This result is in fact a direct consequence of Proposition 5.2 above. More precisely, this proposition immediately implies the following:

Theorem 15.3. Let $d, R_{0}$ be as in Theorem 1.8. There exists effective (explicit) functions $A\left(R_{0}, d\right), B(d)$ such that for every $\left(R_{0}, d\right)$-growth group $(G, S)$ a sequence of at most $B(d)$ operations of the type below reduce the group to the trivial group:

1. Passing to a finite-index subgroup of index bounded by $A\left(R_{0}, d\right)$.

2. Passing to the kernel of a homomorphism to a cyclic group (finite or infinite). 
The proof of this result goes simply by inspecting Proposition 5.2. In each step where 1 occurs, the worst bound on $A\left(R_{0}, d\right)$ comes from possibility (i) in Proposition 5.2, where $A\left(R_{0}, d\right)$ has to be taken as an upper bound for the size of the ball $B_{S}\left(R_{0}^{\exp \left(\exp \left(O(d)^{O(1)}\right)\right)}\right)$. Otherwise, we pass to the finite-index subgroup $G^{\prime}$ appearing in (ii), for which the inclusion $G \subseteq B_{S}\left(R_{0}\right) G^{\prime}$ holds, giving a bound $R_{0}^{d}$ on its index. By Proposition 5.2 the number of operations $B(d)$ performed in this process is obviously $O\left(d^{O(1)}\right)$, thus completing the proof of Theorem 15.3.

To complete the proof of Theorem 15.3, notice that every group which can be reduced to the trivial group using the operations 1 and 2 above is virtually polycyclic in an effective manner, by applying the following lemma as many times as necessary: LEMmA 15.4. If a group $G$ has a subgroup $G^{\prime}$ of index at most $I$ which contains a polycyclic normal subgroup of index at most $J$, then $G$ has a polycyclic normal subgroup of index at most $(I \cdot J)$ !.

Proof. By hypothesis, $G$ has a polycyclic subgroup $G^{\prime \prime}$ of index at most $I J$. The group $G$ acts by left multiplication on the quotient set $G / G^{\prime \prime}$; the stabilizer $H$ of this action then has index at most $(I J)$ !, and is a normal subgroup of $G$ that is contained in $G^{\prime \prime}$. Since any subgroup of a polycyclic group is polycyclic, the claim follows.

REMARK 15.5. Of course, only type 1 steps contribute to the computation of the total index (this makes the computation effective; unfortunately, the lack of reasonable control over the size of the finite quotients appearing in 2 is responsible for our inability to get a similarly effective result concerning the index of a nilpotent subgroup).

Finally, the following observation shows that torsion is the only obstacle at this point to obtaining a fully effective version of Gromov's theorem, an issue which will be shortly discussed in more detail over the next subsection.

Corollary 15.6 (to Theorem 1.8). Retain the assumptions of Theorem 1.8, but assume further that $G$ is torsion free. Then a finite-index nilpotent subgroup as in the theorem can be found with index $q$ satisfying

$$
\log \log \log q<C^{d}
$$

where $C$ is an absolute (effective) constant.

This follows immediately from Corollary 1.12 and a result of Auslander and Schenkman - see [Gr, p.71] and the reference therein.

15.7 Towards full effectivization of Theorem 1.8. For the purpose of full quantification one must forego the compactness argument in section 3 that allowed us to reduce Theorem 1.8 to Theorem 3.2. Instead, one must replace "virtually $r$-nilpotent" by something more like " $(K, R, s, D)$-virtually nilpotent" throughout the arguments, making sure to keep the bounds on $K, R$ effective.

Of the three components of the proof in section 5, the first two (Lemma 5.1 and Proposition 5.2) are already completely effective (and do not use the notion of virtual $r$-nilpotency). The main issue, therefore, is to locate an effective version of 
Proposition 5.3. This proposition is applied about $O(d)$ times during the induction on the growth order $d$ in the proof of Theorem 1.8, so any deterioration in the $K, R$ constants occurring in that proposition will need to be iterated $O(d)$ times to obtain the final bound on $K\left(R_{0}, d\right)$. The arguments in section 11, in which Proposition 5.3 is reduced to Proposition 11.2, are also quite effective, in the sense that any effective version of the latter can be converted by a routine modification of the arguments in that section to a quantitative version of the former. But as before, the deterioration of the bounds will worsen due to the induction on the step $l$ and the dimension $m$ appearing in the arguments in that section (more precisely, the bounds in Proposition 5.3 will basically be a $O(1)^{d}$-fold iteration of the bounds in Proposition 11.2).

The key proposition in section 12, namely Proposition 12.1, is already effective. The key proposition in section 13, namely Proposition 13.1, can also be made effective without much difficulty, for instance by using the Kronecker approximation theorem to quantitatively approximate a vector by one with rational coefficients, and using Cramer's rule to bound all the vectors that are constructed from linear algebra (e.g. locating an integer vector in the null space of a matrix with integer coefficients). To deal with various denominators in Cramer's rule, one does need to obtain a quantitative lower bound on the difference $\left|\lambda_{i}-\lambda_{j}\right|$ between two eigenvalues of a matrix $T$ with integer coefficients, but this can be accomplished by using Galois theory to observe that the product $\prod_{\lambda_{i} \neq \lambda_{j}}\left(\lambda_{i}-\lambda_{j}\right)$ of all these eigenvalue gaps is a non-zero rational integer, and in particular has magnitude at least 1 . We omit the details.

The only remaining parts of the argument which need more careful attention are those in section 14, as it is here that one truly begins to exploit such qualitative notions as Hirsch length and torsion freeness.

Recall that a group $G=(G, S)$ is virtually $r$-nilpotent if it contains a finiteindex nilpotent subgroup $G^{\prime}=\left(G^{\prime}, S^{\prime}\right)$ which is torsion free of Hirsch length $r$ (and thus step at most $r$ ). The notion of finite index can be made quantitative using the concept of a $(K, R)$-subgroup as defined in Definition 1.3. Nilpotency of a given step is also a quantitative concept (being nilpotent of step $s$ is equivalent to the $s$-fold commutators of the generating set $S^{\prime}$ vanishing). However, torsion freeness cannot be verified using only finitely many group operations on the generators and so should not be considered as quantitative. (For instance, the additive group $\mathbf{Z} / N \mathbf{Z}$ with generating set $\{-1,+1\}$ has torsion, in contrast to the torsion-free group $\mathbf{Z}$ with the same set of generators, but it is only possible to distinguish the two groups after performing at least $O(\log N)$ group operations on the generators, which is unbounded as $N \rightarrow \infty$.) This is a genuine problem in the proof, as torsion freeness is used crucially in section 14 in order to bound $\left\|T^{n} h\right\|_{S^{\prime \prime \prime}}$ from below using the norm of a projection of $T^{n} h$ to a free abelian group $\mathbf{Z}^{d_{i}}$.

It is thus natural to seek finitary substitutes for the concept of being torsion-free. Call a abelian group $G=(G,+)$ generated by a set $S=\left\{ \pm e_{1}, \ldots, \pm e_{D}\right\}$ of generators $e_{1}, \ldots, e_{D} M$-torsion-free for some $M \geq 1$ if the sums $\left\{n_{1} e_{1}+\ldots+n_{D} e_{D}\right.$ : $\left.\left|n_{1}\right|+\ldots+\left|n_{D}\right| \leq M\right\}$ that make up $B_{S}(M)$ are all distinct. This property, in 
contrast to the qualitative property of being torsion-free, can be verified in finite time using a finite number of group operations on generators, and so we consider this a quantitative property. A key ingredient in the proof that any finitely generated nilpotent group has a finite-index torsion-free subgroup is that same result for abelian groups. We now give a quantitative version of this fact. It is convenient to extend the asymptotic notation $X \ll Y$ by allowing the constants to depend on additional parameters, indicated by subscripts on the $\ll$ symbol; for instance, $X \ll_{D} Y$ means that $X \leq C(D) Y$ for some quantity $C(D)$ depending only on $D$. (Of course, to get effective bounds at the end of the day, it is important to ensure that all implied constants such as $C(D)$ depend in an effective fashion on parameters such as $D$.)

LEmmA 15.8 (Quantitative location of an $M$-torsion-free group). Let $G=(G, S,+)$ be a finitely generated abelian group with $D$ generators $S=\left\{ \pm e_{1}, \ldots, \pm e_{D}\right\}$, and let $F: \mathbf{R}^{+} \rightarrow \mathbf{R}^{+}$be an arbitrary function. Then there exists an integer $1 \leq M \ll_{F, D} 1$ and a $(M, 1)$-subgroup $\left(G^{\prime}, S^{\prime}\right)$ of $G$ with $S^{\prime}=\left\{ \pm f_{1}, \ldots, \pm f_{D^{\prime}}\right\}$ for some $1 \leq D^{\prime} \leq D$, such that $\left(G^{\prime}, S^{\prime}\right)$ is $F(M)$-torsion-free.

Proof (Sketch). We use the "rank reduction argument". We begin by initializing $\left(G^{\prime}, S^{\prime}\right)$ equal to $(G, S)$ and initializing $M:=1$, then $\left(G^{\prime}, S^{\prime}\right)$ is already an $(M, 1)$ subgroup of $(G, S)$. If $\left(G^{\prime}, S^{\prime}\right)$ is $F(M)$-torsion-free then we are done. If not, write $S^{\prime}=\left\{ \pm f_{1}, \ldots, \pm f_{D^{\prime}}\right\}$ for some $D^{\prime}$ (which is initially equal to $D$ ). Because of the failure of $\left(G^{\prime}, S^{\prime}\right)$ to be $F(M)$-torsion-free, we must have a non-trivial dependence

$$
n_{1} f_{1}+\ldots+n_{D^{\prime}} f_{D^{\prime}}=0
$$

where $0<\left|n_{1}\right|+\ldots+\left|n_{D^{\prime}}\right| \leq 2 F(M)$. Without loss of generality we may take $n_{D^{\prime}}$ to have the largest magnitude, and in particular be non-zero. Then we see that the subgroup $\left(G^{\prime \prime}, S^{\prime \prime}\right)$ of $\left(G^{\prime}, S^{\prime}\right)$ generated by $S^{\prime \prime}:=\left\{ \pm f_{1}, \ldots, \pm f_{D^{\prime}-1}\right\}$ has finite index (indeed, the index is at most $\left|n_{D^{\prime}}\right|$ ); more quantitatively, one can show that $\left(G^{\prime \prime}, S^{\prime \prime}\right)$ is a $\left(O\left(D^{\prime} F(M)\right), 1\right)$-subgroup of $\left(G^{\prime}, S^{\prime}\right)$, which by Lemma 4.1 implies that $\left(G^{\prime \prime}, S^{\prime \prime}\right)$ is a $\left(M^{\prime}, 1\right)$-subgroup of $(G, S)$ for some $M^{\prime}=O(D M F(M))^{O(1)}$. One then replaces $\left(G^{\prime}, S^{\prime}\right), D^{\prime}, M$ by $\left(G^{\prime \prime}, S^{\prime \prime}\right), D^{\prime \prime}, M^{\prime}$ respectively, and iterates this procedure. Since the rank $D^{\prime}$ starts at $D$ and decreases by one at each stage, this algorithm terminates in at most $D$ steps, and the claim follows.

REMARK 15.9. The eventual bound on $M$ is essentially a $D$-fold iteration of $F$. Unfortunately, in applications $F$ has to be quite a rapidly growing function (exponential or worse), which leads to quite poor bounds, especially after the induction loops on the growth order $d$, the solvability index $l$, and the dimension $D$ that appear in the proof are carried out. It is thus of interest to reduce the dependence on the torsion-free property (and also to reduce the length of the induction loops) in order to improve the bounds.

In a similar vein to the above discussion, it is possible to define a quantitative notion of an " $M$-torsion-free nilpotent group", and establish a quantitative version of the fact that every finitely generated nilpotent group contains a finite-index torsionfree subgroup, which roughly speaking asserts that for any given $F$, any $(s, D)$ - 
nilpotent subgroup will have an $(M, M)$-subgroup which is $F(M)$-torsion-free for some $M \ll_{F, s, D} 1$. This will turn out to be a usable quantitative substitute for the qualitative notion of torsion freeness if $F$ is chosen to be sufficiently rapidly growing. Indeed, the various vectors in $\mathbf{Z}^{D}$ which appear in the arguments in section 14 will have norm bounded by some effective function of the parameter $M$ (thanks to quantitative versions results such as Proposition 13.1), and as long as $F(M)$ is much larger than the norm of these vectors, then the $M$-torsion-free nilpotent groups involved will behave "as if" they are genuinely torsion free for the purposes of the computations being performed. For instance, it would suffice to take $F(M):=$ $\exp \left(\exp \left(\exp \left((C d l M)^{C}\right)\right.\right.$ for some large absolute constant $C$. If one does this, then the final bounds obtained on $K\left(R_{0}, d\right)$ are essentially an Ackermann function of $R_{0}$ and $O(1)^{d}$.

\section{References}

[CM] T. Colding, W. Minicozzi, II, Harmonic functions on manifolds, Ann. Math. 146 (1997), 725-747.

[D] E. Dobrowolski, On a question of Lehmer and the number of irreducible factors of a polynomial, Acta Arith. 34:4 (1979), 391-401.

[DrW1] L. VAN DEN DRIES, A.J. Wilkie, Gromov's theorem on groups of polynomial growth and elementary logic, J. Alg. 89 (1984), 349-374.

[DrW2] L. van den Dries, A.J. Wilkie, An effective bound for groups of linear growth, Arch. Math. (Basel) 42:5 (1984), 391-396.

[G1] R.I. Grigorchuk, On the Hilbert-Poincaré series of graded algebras that are associated with groups (Russian), Mat. Sb. 180:2 (1989), 207-225.

[G2] R.I. GRIGORChuk, Degrees of growth of finitely generated groups and the theory of invariant means, Math. USSR Izv. 25:2 (1985), 259-300; Russian original: Izv. Akad. Nauk SSSR Sr. Mat. 48:5 (1984), 939-985.

[Gr] M. Gromov, Groups of polynomial growth and expanding maps, Inst. Hautes Études Sci. Publ. Math. 53 (1981), 53-73.

$[\mathrm{J}] \quad$ F. John, Extremum problems with inequalities as subsidiary conditions, Studies and Essays presented to R. Courant on his 60th birthday, Interscience Publishers Inc., New York, NY (1948), 187-204.

[K] M. Kapovich, Hyperbolic Manifolds and Discrete Groups, Progress in Mathematics 183, Birkhäuser Boston MA (2001).

[KiSV] A.Y. Kitaev, A.H. Shen, M.N. Vyulyi, Classical and Quantum Computation, Graduate Studies in Mathematics 47, American Mathematical Society, Rhode Island (2002).

[Kl] B. KLEINER, A new proof of Gromov's theorem on groups of polynomial growth, Jour. of the AMS, to appear.

[KoS] N. Korevaar, R. Schoen, Global existence theorems for harmonic maps to nonlocally compact spaces, Comm. Anal. Geom. 5 (1996), 333-387.

[Kr] L. Kronecker, Zwei Sätze über Gleichungen mit ganzzahligen Coefficienten, J. für riene und angew. Math. 53 (1857), 173-175.

[L] M. LAzArd, Groupes analytiques $p$-adiques, Inst. Hautes Études Sci. Publ. Math. 26 (1965), 389-603. 
[LeM] J. LeE, Y. MAKARYChev, Eigenvalue multiplicity and volume growth, preprint.

[LeP] J. LEE, Y. PEREs, Harmonic maps on amenable groups and a diffusive lower bound for random walks, preprint.

[Li] P. LI, Harmonic sections of polynomial growth, Math. Res. Lett. 4 (1997), 35-44.

[LuM] A. Lubotzky, A. Mann, Powerful $p$-groups. II. $p$-adic analytic groups, J. Algebra 105:2 (1987), 506-515.

[LuMS] A. Lubotzky, A. Mann, D. Segal, Finitely generated groups of polynomial subgroup growth, Israel J. Math. 82:1 (1993), 363-371.

[LuPS] A. Lubotzky, L. Pyber, A. Shalev, Discrete groups of slow subgroup growth, Israel J. Math. 96:B (1996), 399-418.

[M1] A.I. MAL'CEv, Nilpotent torsion-free groups (Russian), Izv. Akad. Nauk SSSR. Ser. Mat. 13 (1949), 201-212.

[M2] A.I. MAL'CEV, Two remarks on nilpotent groups (Russian), Mat. Sb. N.S. 79 (1955), 567-572.

[Mi1] J. Milnor, Growth of finitely generated solvable groups, J. Diff. Geom. 2 (1968), $447-449$

[Mi2] J. Milnor, A note on curvature and fundamental group, J. Diff. Geom. 2 (1968), $1-7$.

[Mo] N. MoK, Harmonic forms with values in locally compact Hilbert bundles, Proceedings of the Conference in Honor of Jean-Pierre Kahane (Orsay 1993), Special Issue (1995), 433-454.

[S] D. Segal, The finite images of finitely generated groups, Proc. London Math. Soc. (3) 82:3 (2001), 597-613.

[Sh] Y. Shalom, The growth of linear groups, J. Algebra 199:1 (1998), 169-174.

[ShT] Y. Shalom, T. TaO, On Kleiner's proof of Gromov's theorem and Lipschitz harmonic functions, in preparation.

[T1] T. TAO, Product set estimates for non-commutative groups, preprint.

[T2] T. TAO, Freiman's theorem for solvable groups, preprint.

[Ti] J. Tits, Free subgroups in linear groups, J. Algebra 20 (1972), 250-270.

[W] J. WoLF, Growth of finitely generated solvable groups and curvature of Riemannian manifolds, J. Diff. Geom. 2 (1968), 421-446.

Yehuda Shalom, Department of Mathematics, UCLA, Los Angeles, CA 90095-1555, USA shalom@math.ucla.edu

Terence TAO, Department of Mathematics, UCLA, Los Angeles, CA 90095-1555, USA

tao@math.ucla.edu

Received: October 27, 2009

Accepted: April 12, 2010

Open Access This article is distributed under the terms of the Creative Commons Attribution Noncommercial License which permits any noncommercial use, distribution, and reproduction in any medium, provided the original author(s) and source are credited. 